\title{
Correlating the anomalous results in $b \rightarrow s$ decays with inert Higgs doublet dark matter and the muon $g-2$
}

\author{
Basabendu Barman, ${ }^{*}$ Debasish Borah, ${ }^{\dagger}$ Lopamudra Mukherjee, ${ }^{\ddagger}$ and Soumitra Nandi ${ }^{\S}$ \\ Indian Institute of Technology, North Guwahati, Guwahati 781039, Assam, India
}

(Received 16 May 2019; revised manuscript received 27 August 2019; published 5 December 2019)

\begin{abstract}
In this article, we have considered an extension of the inert Higgs doublet model with $S U(2)_{L}$ singlet vectorlike fermions. Our model is capable of addressing some interesting anomalous results in $b \rightarrow s \ell^{+} \ell^{-}$ decays [like $R\left(K^{(*)}\right)$ ] and in muon $(g-2)$. Apart from explaining these anomalies, and being consistent with other flavor data, the model satisfies relevant constraints in the dark matter sector, while remaining within the reach of ongoing direct detection experiments. The model also produces signatures at the large hadron collider (LHC) with final states comprised of dilepton, dijet and missing energy, providing signals to be probed at higher luminosity.
\end{abstract}

DOI: $10.1103 /$ PhysRevD.100.115010

\section{INTRODUCTION}

The low energy observables in $B$ decays and $B_{q}-\overline{B_{q}}$ $(q=d, s)$ mixings play an important role in the indirect detection of new physics (NP). In this regard, the flavor changing neutral current (FCNC) processes, such as $b \rightarrow s$, are unique in a sense that in the standard model (SM) they contribute at the loop level thereby keeping their contributions suppressed, in general. For the past couple of years, the semileptonic decays $b \rightarrow s \ell^{+} \ell^{-}(\ell=\mu, e)$ have gotten a lot of attention. The observed ratios of the exclusive branching fractions such as $R\left(K^{(*)}\right)=\mathcal{B}(B \rightarrow$ $\left.K^{(*)} \mu^{+} \mu^{-}\right) / \mathcal{B}\left(B \rightarrow K^{(*)} e^{+} e^{-}\right)$have shown anomalous behaviors with the measured values deviating from their respective SM expectations. The LHCb collaboration has measured $[1,2]$

$$
R(K)=0.846_{-0.054-0.014}^{+0.060+0.016}
$$

in the bin with dilepton mass squared

$$
q^{2} \in[1.1,6] \mathrm{GeV}^{2} \text {, }
$$

and

\footnotetext{
*bb1988@iitg.ac.in

†dborah@iitg.ac.in

*mukherjeelopa@iitg.ac.in

$\S_{\text {soumitra.nandi@iitg.ac.in }}$
}

Published by the American Physical Society under the terms of the Creative Commons Attribution 4.0 International license. Further distribution of this work must maintain attribution to the author(s) and the published article's title, journal citation, and DOI. Funded by SCOAP.
$R\left(K^{*}\right)= \begin{cases}0.660_{-0.070}^{+0.110} \pm 0.024, & q^{2} \in[0.045,1.1] \mathrm{GeV}^{2}, \\ 0.685_{-0.069}^{+0.113} \pm 0.047, & q^{2} \in[1.1,6] \mathrm{GeV}^{2}\end{cases}$

The corresponding SM predictions are, respectively, $R(K)=1.0004(8)$, and

$R\left(K^{*}\right)= \begin{cases}0.920 \pm 0.007, & q^{2} \in[0.045,1.1] \mathrm{GeV}^{2}, \\ 0.996 \pm 0.002, & q^{2} \in[1.1,6] \mathrm{GeV}^{2},\end{cases}$

the details of which can be found in [3,4]. In our analysis, we have not included the very recent results on $R\left(K^{(*)}\right)$ by Belle collaboration $[5,6]$. This is because the data has large error bars, making a meaningful comparison of the results difficult. Therefore, the observed data indicate a possible violation of lepton universality. There has been plenty of analysis on the NP explanations of the observed discrepancies, which we are not going to elaborate on here. In order to explain the observed discrepancies, one needs to develop a new mechanism that will generate lepton universality violation either at the tree level or via loops.

Among the other important observables, the anomalous magnetic moment of muon shows deviation between theory and experiment. Particle magnetic moments are good probes of physics beyond the SM, and the similar study could shed light on our understanding of quantum electrodynamics (QED) and the SM. The anomalous magnetic moment of muon has been measured very precisely while it has also been predicted in the SM to a great accuracy. The muon anomalous magnetic moment is defined as

$$
a_{\mu} \equiv \frac{g_{\mu}-2}{2}
$$


which includes the quantum loop effects, and parametrizes the small calculable deviation from $g_{\mu}=2$ (Lande's $g$ factor). The SM contributions to $a_{\mu}$ can be expressed as

$$
a_{\mu}^{\mathrm{SM}}=a_{\mu}^{\mathrm{QED}}+a_{\mu}^{\mathrm{EW}}+a_{\mu}^{\mathrm{Had}},
$$

where $a_{\mu}^{\mathrm{QED}}, a_{\mu}^{\mathrm{EW}}$ and $a_{\mu}^{\mathrm{Had}}$ are the contributions from QED loops, electroweak loops and hadronic loops respectively. This quantity has been measured very accurately and at present the difference between the predicted and the measured value is given by

$$
\Delta a_{\mu}=a_{\mu}^{\exp }-a_{\mu}^{\mathrm{SM}}=26.8(7.6) \times 10^{-10},
$$

which shows there is still room for NP beyond the SM (for details see [7]). In this study, we will look for a NP model which is capable of addressing simultaneously both of the above-mentioned excesses.

On the other hand, dark matter (DM) has been understood to be present in a significant amount in the present Universe, roughly 5 times the abundance of ordinary baryonic matter [8]. The present dark matter abundance, measured by Planck [8] is often quoted as

$$
\Omega_{\mathrm{DM}} h^{2}=\frac{\rho_{\mathrm{DM}}}{\rho_{\mathrm{c}}} h^{2}=0.1198 \pm 0.0015,
$$

where $h=H_{0} /\left(100 \mathrm{~km} \mathrm{~s}^{-1} \mathrm{Mpc}^{-1}\right), \rho_{\mathrm{DM}}$, and $\rho_{\mathrm{c}}=\frac{3 H_{0}^{2}}{8 \pi G}$ are, respectively, the present day normalized Hubble expansion rate $\left(H_{0}\right)$, DM density, and the critical density of the Universe, whereas $G$ is the universal constant of gravity. Such cosmological evidences are also complemented by astrophysical evidences suggesting the presence of nonluminous and nonbaryonic matter components in the Universe [9-11].

In the SM, we do not have a suitable DM candidate which satisfies the requirements as given in [12]. This has led to several beyond the standard model (BSM) proposals which can successfully explain DM in the Universe. Among different BSM prescriptions, the paradigm with a generic weakly interacting massive particle (WIMP) is well motivated. In such scenarios, the DM particle has mass and interactions typically around the electroweak ballpark and can give rise to the correct dark matter relic abundance, a remarkable coincidence often referred to as the WIMP Miracle (see, e.g., [13]). Since WIMP dark matter scenarios involve additional physics around the electroweak scale, it is tempting to speculate if the same new physics can have plausible explanations for the observed flavor anomalies like $R\left(K^{(*)}\right), a_{\mu}$ mentioned earlier. Within such a unified framework, one needs to find out the allowed NP parameter space consistent with flavor data as well as the requirements for a DM candidate. Also, it is necessary to check that the required NP parameter spaces are consistent with all the other relevant measurements which are not anomalous. There have been several attempts along this direction, some of which can be found in [14-18] and references therein. Apart from being consistent with all these observations, it is also important for such a scenario to be predictive at different experiments like direct detection of dark matter, collider searches and so on.

In a model independent analysis [19], by considering an effective theory framework, it has been shown that the deficit in the lepton universality ratio $R\left(K^{(*)}\right)$ can be best explained by the set of the operators $\mathcal{O}_{9}^{\ell}=\left[\bar{b} \gamma_{\mu} P_{L} s\right]\left[\bar{l} \gamma^{\mu} l\right]$ and $\mathcal{O}_{10}^{\ell}=\left[\bar{b} \gamma_{\mu} P_{L} s\right]\left[\bar{l} \gamma^{\mu} \gamma_{5} l\right]$. Therefore, the NP models under consideration should give rise to these four-Fermi interactions either via tree or loop level diagrams for the process $b \rightarrow s \ell \ell$. Here, we consider the inert Higgs doublet model (IDM), which is a simple extension of the SM by an additional scalar field $\Phi_{2}$ transforming as doublet under $S U(2)_{L}$ gauge symmetry and has hypercharge $Y=1$. The model has been introduced in [20], and later studied extensively by several groups in the context of DM phenomenology [21-30].

In this model, an additional discrete $Z_{2}$ symmetry is introduced in order to prevent the coupling of this scalar field to the SM fermions. Under this $Z_{2}$ symmetry, the additional scalar field transforms as $\Phi_{2} \rightarrow-\Phi_{2}$ whereas all SM fields are even. If the lightest component of $\Phi_{2}$ is electromagnetically neutral, it can be stable and hence a good DM candidate. Being inert in nature, IDM will not contribute to the decay $b \rightarrow s \ell \ell$. Hence, we have extended this model by considering three generations of vectorlike $S U(2)_{L}$ singlet down-type quarks and charged leptons, odd under the $Z_{2}$ symmetry so that they can couple to the SM quarks and leptons only through the inert scalar doublet. The lightest component of $\Phi_{2}$ remains the lightest $Z_{2}$ odd particle of the model and hence the DM candidate. We have shown that, apart from explaining the DM abundance of the Universe, the model can also explain the observed pattern in $R\left(K^{(*)}\right)$. This model has family nonuniversal Yukawa couplings between $\Phi_{2}$, vectorlike fermions and the SM fermions. Hence, it will contribute to $b \rightarrow s \ell \ell$ at one-loop level. Now, if we consider a hierarchical structure between the Yukawa couplings of electron and muon with the new vectorlike fermions, then we can expect to get $R\left(K^{(*)}\right) \neq 1$. The additional vectorlike fermions can also contribute to the relic abundance, as well as direct detection scattering rates of DM in this model, giving us a complementary probe of the model parameters in both $\mathrm{DM}$ and flavor experiments.

In the pure IDM there exists two mass ranges where DM relic abundance can be satisfied: one in the low mass regime below the $W$ boson mass threshold $\left(M_{\mathrm{DM}}<M_{W}\right)$ and the other around $550 \mathrm{GeV}$ or above. In our extended IDM, there will be additional annihilation channels of DM. Therefore, it is important to rescan the parameter space for both the pure and the extended IDM. The direct detection scattering in pure IDM is primarily mediated by the SM 
Higgs and faces the strongest constraints from the direct detection experiments in the low mass regime. For example, the latest data from the LUX experiment rules out DM-nucleon spin independent cross section above around $2.2 \times 10^{-46} \mathrm{~cm}^{2}$ for DM mass of around $50 \mathrm{GeV}$ [31]. On the other hand, the recently released results from the XENON-1T experiment rule out spin independent WIMPnucleon interaction cross section above $7.7 \times 10^{-47} \mathrm{~cm}^{2}$ for $\mathrm{DM}$ mass of $35 \mathrm{GeV}$ [32]. These strong bounds reduce the allowed DM masses in the low mass regime to a very narrow region near the SM-like Higgs resonance $M_{\mathrm{DM}} \approx m_{h} / 2$. Although the direct detection limits can be somewhat relaxed in the high mass regime $\left(M_{\mathrm{DM}} \gtrsim 550 \mathrm{GeV}\right)$, the production of DM at colliders will be suppressed compared to the low mass regime. In the presence of additional vectorlike quarks, there are additional diagrams which will contribute to the spin independent direct detection cross section. We in fact find that, compared to the pure IDM, the presence of new vectorlike fermions can keep the dark matter direct detection rates closer to the experimental upper bound for some choices of parameters.

The mediators of our model couple to SM quarks and leptons, therefore interesting collider signatures are expected with leptons and/or jets in the final state with missing energy. We study the final states containing $\left(\ell^{+} \ell^{-}+\mathbb{E}_{T}\right),\left(j j+\mathbb{E}_{T}\right)$ and $\left(\ell^{+} \ell^{-}+j j+\mathscr{E}_{T}\right)$ to unravel the model in the large hadron collider (LHC). These final states are already explored in supersymmetry searches, and important constraints have been obtained on the parameter space $[33,34]$. There have also been some studies on collider signatures of pure IDM, e.g., see [35-37]. In our model, we prepare a few benchmark scenarios by choosing points from the new parameter spaces which are allowed by flavor data and overcome bounds from the DM searches. We have predicted the kinematical distributions of our signal events and compared them with the respective SM backgrounds. We find that at the high luminosity LHC the model may be observed for a few benchmark scenarios at more than $5 \sigma$ significance. We also check the perturbative unitarity of the model and find that for the chosen benchmark points the model can remain perturbative up to an energy scale $10^{5}-10^{7} \mathrm{GeV}$.

The paper is organized as follows: in Sec. II we discuss the particle content and possible interactions, followed by the dark matter phenomenology of the model in Sec. III; constraints from muon $(g-2)$ and lepton flavor violating decays are discussed in Sec. IV; contributions in $b \rightarrow s$ transitions are studied in Sec. V; results from DM and flavor analysis are discussed Sec. VI and some benchmark points are also chosen for further collider study; we then discuss the fate of this model at the LHC in Sec. VII, pointing out the possibility of probing it in future higher luminosity; the renormalization group equation (RGE) runnings are discussed in Sec. VIII and finally we summarize in Sec. IX.

\section{IDM WITH VECTORLIKE FERMIONS}

As mentioned earlier, the IDM is an extension of the SM by an additional global discrete $Z_{2}$ symmetry under which a newly incorporated scalar doublet $\Phi_{2}$ transforms as $\Phi_{2} \rightarrow-\Phi_{2}$, while the usual SM fields are even under $Z_{2}$. The requirement of keeping the $Z_{2}$ symmetry unbroken prevents the neutral component of the second Higgs doublet from acquiring a nonzero vacuum expectation value (vev). Since the same discrete symmetry prevents any coupling of $\Phi_{2}$ with the SM fermions, it automatically makes the lightest component of $\Phi_{2}$ stable and hence a good DM candidate. The scalar potential of the model involving the SM Higgs doublet $\Phi_{1}$ and the inert doublet $\Phi_{2}$ can be written as

$$
\begin{aligned}
V\left(\Phi_{1}, \Phi_{2}\right)= & \mu_{1}^{2}\left|\Phi_{1}\right|^{2}+\mu_{2}^{2}\left|\Phi_{2}\right|^{2}+\frac{\lambda_{1}}{2}\left|\Phi_{1}\right|^{4}+\frac{\lambda_{2}}{2}\left|\Phi_{2}\right|^{4} \\
& +\lambda_{3}\left|\Phi_{1}\right|^{2}\left|\Phi_{2}\right|^{2}+\lambda_{4}\left|\Phi_{1}^{\dagger} \Phi_{2}\right|^{2} \\
& +\left\{\frac{\lambda_{5}}{2}\left(\Phi_{1}^{\dagger} \Phi_{2}\right)^{2}+\text { H.c. }\right\} .
\end{aligned}
$$

As the electroweak symmetry has to be broken by the vev of $\Phi_{1}$, we assume $\mu_{1}^{2}<0$. Also, $\mu_{2}^{2}>0$ is assumed so that $\Phi_{2}$ does not acquire a vev. Writing the scalar fields in terms of components and expanding the field $\Phi_{1}$ about the nonzero vev, we have

$$
\Phi_{1}=\left(\begin{array}{c}
0 \\
\frac{v+h}{\sqrt{2}}
\end{array}\right), \quad \Phi_{2}=\left(\begin{array}{c}
H^{ \pm} \\
\frac{H^{0}+i A^{0}}{\sqrt{2}}
\end{array}\right)
$$

in unitary gauge. Here $v$ is the vev of the neutral component of $\Phi_{1}$. After electroweak symmetry breaking, the masses of the physical scalars, at tree level, can be written as

$$
\begin{aligned}
m_{h}^{2} & =\lambda_{1} v^{2}, \\
M_{H^{ \pm}}^{2} & =\mu_{2}^{2}+\frac{1}{2} \lambda_{3} v^{2}, \\
M_{H^{0}}^{2} & =\mu_{2}^{2}+\frac{1}{2}\left(\lambda_{3}+\lambda_{4}+\lambda_{5}\right) v^{2}=M_{H^{ \pm}}^{2}+\frac{1}{2}\left(\lambda_{4}+\lambda_{5}\right) v^{2}, \\
M_{A^{0}}^{2} & =\mu_{2}^{2}+\frac{1}{2}\left(\lambda_{3}+\lambda_{4}-\lambda_{5}\right) v^{2}=M_{H^{ \pm}}^{2}+\frac{1}{2}\left(\lambda_{4}-\lambda_{5}\right) v^{2} .
\end{aligned}
$$

Here $m_{h} \approx 125 \mathrm{GeV}$ is the mass of the SM Higgs, $M_{H^{0}}, M_{A^{0}}$ are the masses of the $C P$ even and $C P$ odd scalars of the inert doublet while $M_{H^{ \pm}}$is the mass of the charged scalar. Without any loss of generality, we consider $\lambda_{5}<0, \lambda_{4}+\lambda_{5}<0$ so that the $C P$ even scalar is the lightest $Z_{2}$ odd particle and hence a stable dark matter candidate.

Apart from the $Z_{2}$ odd scalar doublet $\Phi_{2}$, we consider additional vectorlike charged fermions too, which are odd 
TABLE I. Particle content of the extension of IDM by vectorlike fermions.

\begin{tabular}{lcc}
\hline \hline Particles & $S U(3)_{c} \times S U(2)_{L} \times U(1)_{Y}$ & $Z_{2}$ \\
\hline$Q_{L}=\left(\begin{array}{cc}u_{L} \\
d_{L}\end{array}\right)$ & $\left(3,2, \frac{1}{6}\right)$ & + \\
$u_{R}$ & $\left(3,1, \frac{2}{3}\right)$ & + \\
$d_{R}$ & $\left(3,1,-\frac{1}{3}\right)$ & + \\
$L_{L}=\left(\begin{array}{c}\nu_{L} \\
e_{L}\end{array}\right)$ & $\left(1,2,-\frac{1}{2}\right)$ & + \\
$e_{R}$ & $(1,1,-1)$ & + \\
$\Phi_{1}$ & $\left(1,2, \frac{1}{2}\right)$ & + \\
$\Phi_{2}$ & $\left(1,2, \frac{1}{2}\right)$ & - \\
$D_{L, R}$ & $\left(3,1,-\frac{1}{3}\right)$ & - \\
$E_{L, R}$ & $(1,1,-1)$ & - \\
\hline \hline
\end{tabular}

under the same $Z_{2}$ symmetry. The particle content of the model is shown in Table I. Here $D$ is the down-type vectorlike quark and $E$ is the vectorlike lepton. This allows the coupling of the inert doublet scalar with the SM fermions through the vectorlike fermion portal. The relevant Yukawa Lagrangian can be written as

$$
\begin{aligned}
\mathcal{L}= & \left(y_{u}\right)_{i j} \bar{Q}_{i} \tilde{\Phi}_{1} u_{R j}+\left(y_{d}\right)_{i j} \bar{Q}_{i} \Phi_{1} d_{R j}+\left(y_{e}\right)_{i j} \bar{L}_{i} \Phi_{1} e_{R j} \\
& +\left(\lambda^{D}\right)_{i j} \bar{Q}_{i} \Phi_{2} D_{R j}+\left(\lambda^{E}\right)_{i j} \bar{L}_{i} \Phi_{2} E_{R j} \\
& +M_{D_{i}} \bar{D}_{L i} D_{R i}+M_{E_{i}} \bar{E}_{L i} E_{R i}+\text { H.c. }
\end{aligned}
$$

where $\tilde{\Phi}_{1,2}=i \tau_{2} \Phi_{1,2}^{*}$ and $\lambda^{E, D}$ are the Yukawa couplings associated with the vector fermion interactions. Also, $i$ and $j$ are the generation indices.

We are working in a basis where the vectorlike fermion fields are diagonal. Also, the SM fields can be rotated to the corresponding mass basis. In our analysis, in the quark sector the phenomenologically relevant new interactions involve only SM down-type quarks. Therefore, while rotating the SM quark fields, in principle, we can choose a basis where down-type quarks are diagonal. In such a case, there will not be any changes in the definitions of our new Yukawas. On the other hand, if we work in a basis where SM up-type quarks are diagonal, then there will be additional flavor mixing of Cabibbo-Kobayashi-Maskawa type in the relevant interactions. These mixings will redefine our Yukawas $\left(\lambda_{i j}^{D}\right)$ in Eq. (10) to say $\lambda_{i j}^{D \prime}$. However, phenomenologically this will not add any new information in our analysis. The similar analysis could be done with $\lambda_{i j}^{D \prime}$.

In the lepton sector, the new charge current interactions involving neutrinos are not phenomenologically relevant in our analysis. The phenomenologically relevant new interactions involve only charged lepton Yukawas. Therefore, the additional vectorlike charged fermions do not contribute to the generation of light neutrino mass. Now, even if neutrino masses are generated by some new physics mechanism, the corresponding mixing angles will not enter in any of the observables or physical processes discussed in this work.

\section{DARK MATTER PHENOMENOLOGY}

In this section we discuss the DM phenomenology of this model in terms of relic density and direct search bounds. We divide the discussion into the following two subsections.

\section{A. Relic abundance of DM}

For a single component DM, the relic abundance can be obtained by solving the Boltzmann equation (BEQ):

$$
\frac{d n_{\mathrm{DM}}}{d t}+3 H n_{\mathrm{DM}}=-\langle\sigma v\rangle\left(n_{\mathrm{DM}}^{2}-\left(n_{\mathrm{DM}}^{\mathrm{eq}}\right)^{2}\right),
$$

where $n_{\mathrm{DM}}$ is the number density of the DM particle, $n_{\mathrm{DM}}^{\mathrm{eq}}$ is the equilibrium number density and $H$ is the Hubble expansion rate. The thermally averaged annihilation cross section $\langle\sigma v\rangle$ can be expanded in powers of (nonrelativistic) velocity as $\langle\sigma v\rangle=a+b v^{2}+\cdots$, where the first term corresponds to $s$-wave, the second term corresponds to $p$-wave and so on. Under this approximation, BEQ can be solved numerically to find the present day relic density of the DM $[38,39]$ :

$$
\Omega_{\mathrm{DM}} h^{2} \approx \frac{1.04 \times 10^{9} x_{F}}{M_{\mathrm{Pl}} \sqrt{g_{*}}\left(a+3 b / x_{F}\right)},
$$

where $x_{F}=M_{\mathrm{DM}} / T_{F}, T_{F}$ is the freeze-out temperature, $M_{\mathrm{DM}}$ is the mass of dark matter, $g_{*}$ is the total number of relativistic degrees of freedom (d.o.f.) at the time of freeze-out $(\sim 106)$ and $M_{\mathrm{Pl}} \approx 2.4 \times 10^{18} \mathrm{GeV}$ is the reduced Planck mass. WIMPs generally freeze out at $x_{F} \approx\{20-30\}$. Generically, $x_{F}$ can be obtained from the relation

$$
x_{F}=\ln \frac{0.038 g M_{\mathrm{Pl}} M_{\mathrm{DM}}\langle\sigma v\rangle}{g_{*}^{1 / 2} x_{F}^{1 / 2}},
$$

which is derived from the equality condition of DM interaction rate $\Gamma=n_{\mathrm{DM}}\langle\sigma v\rangle$ with the rate of expansion of the Universe $H \approx g_{*}^{1 / 2} \frac{T^{2}}{M_{\mathrm{Pl}}}$ (i.e., the freeze-out condition).

For all practical purposes, one can obtain the approximate analytical solution for relic density as [40]

$$
\Omega_{\mathrm{DM}} h^{2} \approx \frac{3 \times 10^{-27} \mathrm{~cm}^{3} \mathrm{~s}^{-1}}{\langle\sigma v\rangle} .
$$

The thermally averaged annihilation cross section $\langle\sigma v\rangle$ is given by [41] 


$$
\begin{aligned}
\langle\sigma v\rangle= & \frac{1}{8 m^{4} T K_{2}^{2}\left(M_{\mathrm{DM}} / T\right)} \int_{4 M_{\mathrm{DM}}^{2}}^{\infty} \sigma\left(s-4 M_{\mathrm{DM}}^{2}\right) \\
& \times \sqrt{ } s K_{1}(\sqrt{ } s / T) d s,
\end{aligned}
$$

where $K_{i}$ 's are modified Bessel functions of order $i$.

In the presence of coannihilation, the effective cross section can be expressed as [42]

$$
\begin{aligned}
\sigma_{\mathrm{eff}} & =\sum_{i, j}^{N}\left\langle\sigma_{i j} v\right\rangle r_{i} r_{j} \\
& =\sum_{i, j}^{N}\left\langle\sigma_{i j} v\right\rangle \frac{g_{i} g_{j}}{g_{\mathrm{eff}}^{2}}\left(1+\Delta_{i}\right)^{3 / 2}\left(1+\Delta_{j}\right)^{3 / 2} e^{\left(-x_{F}\left(\Delta_{i}+\Delta_{j}\right)\right)},
\end{aligned}
$$

where $x_{F}=\frac{M_{\mathrm{DM}}}{T_{F}}$ and $\Delta_{i}=\frac{m_{i}-M_{\mathrm{DM}}}{M_{\mathrm{DM}}}$, where the masses of the heavier components of the inert Higgs doublet are denoted by $m_{i}$. The total number of effective d.o.f. is given by

$$
g_{\mathrm{eff}}=\sum_{i=1}^{N} g_{i}\left(1+\Delta_{i}\right)^{3 / 2} e^{-x_{F} \Delta_{i}} .
$$

The thermally averaged cross section then reads

$$
\begin{aligned}
\left\langle\sigma_{i j} v\right\rangle= & \frac{x_{F}}{8 m_{i}^{2} m_{j}^{2} M_{\mathrm{DM}} K_{2}\left(\left(m_{i} / M_{\mathrm{DM}}\right) x_{F}\right) K_{2}\left(\left(m_{j} / M_{\mathrm{DM}}\right) x_{F}\right)} \\
& \times \int_{\left(m_{i}+m_{j}\right)^{2}}^{\infty} d s \sigma_{i j}\left(s-2\left(m_{i}^{2}+m_{j}^{2}\right)\right) \\
& \times \sqrt{s} K_{1}\left(\sqrt{s} x_{F} / M_{\mathrm{DM}}\right) .
\end{aligned}
$$

The relic density can be again computed by the approximate analytical solution:

$$
\Omega_{\mathrm{DM}} h^{2}=\frac{2.4 \times 10^{-10}}{\sigma_{\text {eff }}} \mathrm{GeV}^{-2} .
$$

In the present model, discussed in the previous section, we consider one of the neutral components of the scalar doublet $\Phi_{2}$, namely $H^{0}$, as the DM candidate for our analysis. This is similar to the inert doublet model of dark matter discussed extensively in the literature [21-27,30].
In the low mass regime $\left(M_{H^{0}} \equiv M_{\mathrm{DM}} \leq M_{W}\right)$, the annihilation of DM to the SM fermions (through s-channel Higgs mediation) dominates over other channels. As pointed out in [24], the annihilation $H^{0} H^{0} \rightarrow W W^{*} \rightarrow W f \bar{f}^{\prime}$ also plays a role in the $M_{\mathrm{DM}} \leq M_{W}$ region. Depending on the mass differences $M_{H^{ \pm}}-M_{H^{0}}\left(\equiv \Delta M_{H^{ \pm}}\right), M_{A^{0}}-M_{H^{0}}\left(\equiv \Delta M_{A^{0}}\right)$, coannihilation of $H^{0}, H^{ \pm}$and $H^{0}, A^{0}$ become important in determining the relic abundance of the DM. Typically, when the heavier components of the inert scalar doublet have masses close to the DM mass, they can be thermally accessible at the epoch of DM freeze-out. Therefore, the annihilation cross section of DM in such a case gets additional contributions from coannihilations between the DM and the heavier components of the scalar doublet $\Phi_{2}$.

\section{B. Dark matter direct search}

As mentioned earlier, there are severe constraints on spin independent DM-nucleon scattering rates from ongoing experiments [31,32]. In the pure IDM, the tree level DMnucleon elastic scattering can arise through the SM Higgs mediation and the current bounds on direct detection cross section can rule out some portion of the parameter space satisfying relic especially in the low mass regime $M_{\mathrm{DM}} \approx$ $m_{h} / 2$ where bounds are stronger. The elastic DM nucleon scattering in the present model gets additional contributions from exotic quark $D$, as depicted in Fig. 1 where the first diagram corresponds to the usual SM Higgs mediated one. The additional contributions will come from the rest of the two diagrams. There is another possible diagram mediated by the $Z$-boson, even in the pure IDM, but that has already been excluded by recent direct search data. Therefore, in order to forbid the $Z$-mediated channel, the mass of $A^{0}$ has to be kept higher than that of $H^{0}$ by a nonzero value, higher than typical kinetic energy $[\mathcal{O}(100 \mathrm{keV})]$ of a DM particle so that $H^{0}$ cannot scatter inelastically into $A^{0}$. The chosen mass splitting in our analysis satisfies this bound as well as the ones from LEP II data [43]. Hence, in this model we have three direct search graphs corresponding to $t$-channel Higgs and exotic quark mediation and another $s$-channel diagram mediated by the vectorlike quark. Due to these additional diagrams, the direct detection rates of the extended IDM can be more promising compared to the pure IDM, as we will discuss later. In the limit of very large
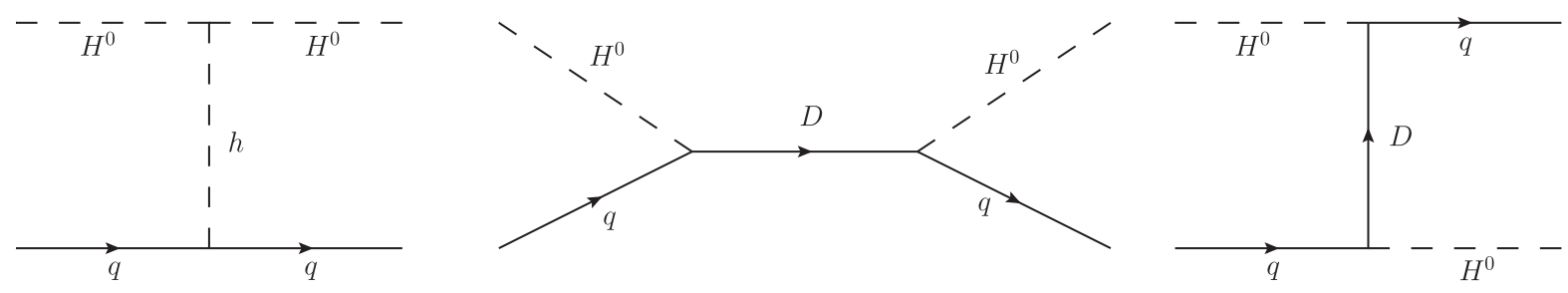

FIG. 1. The Feynman diagrams contributing to the direct search of $H^{0}$. Here, $D$ indicates the contributions from down-type vectorlike quarks (all three generations). 
exotic quark masses or very small couplings of exotic quarks to $\mathrm{DM}$, the direct detection rates will converge towards the ones known for pure IDM.

\section{MUON $(g-2)$ AND THE LEPTON FLAVOR VIOLATION (LFV) DECAYS}

The effective vertex of photon with any charged particle is given by

$$
\begin{aligned}
\bar{u}\left(p^{\prime}\right) e \Gamma_{\mu} u(p)= & \bar{u}\left(p^{\prime}\right)\left[e \gamma_{\mu} F_{1}\left(q^{2}\right)\right. \\
& \left.+\frac{i e \sigma_{\mu \nu} q^{\nu}}{2 m_{f}} F_{2}\left(q^{2}\right)+\cdots\right] u(p) .
\end{aligned}
$$

The factor $g_{\mu} \equiv 2\left(F_{1}(0)+F_{2}(0)\right)$, and the anomalous magnetic moment is given as $a_{\mu} \equiv F_{2}(0) \neq 0$ [since $F_{1}(0)=1$ at all order]. Similarly, the amplitude for the LFV decays $\ell_{i} \rightarrow \ell_{j} \gamma$ can be written as

$$
M_{\gamma}=\bar{u}_{\ell_{j}}\left(p^{\prime}\right)\left[A_{L} q^{2} \gamma_{\mu} P_{L}+i A_{R} m_{\ell_{i}} \sigma_{\mu \nu} q^{\nu} P_{R}\right] u_{\ell_{i}}(p) .
$$

The associated branching fraction can be expressed as

$$
\mathcal{B}\left(\ell_{i} \rightarrow \ell_{j} \gamma\right)=\frac{\alpha \tau_{\ell_{i}}}{4} m_{\ell_{i}}^{5} A_{R}^{2},
$$

where $\tau_{\ell_{i}}$ is the lifetime of the lepton $\ell_{i}$ and $\alpha=1 / 137$ is the fine structure constant.

In our model, the leading contributions in $a_{\mu}$ and the LFV decays like $\tau \rightarrow \mu \gamma, \mu \rightarrow e \gamma$ and $\tau \rightarrow e \gamma$ are obtained from the diagrams in Fig. 2. In the loop, we have either $H^{0}$ or $A^{0}$ and the vectorlike lepton $E$ (which could be either of $E_{1}, E_{2}$ or $E_{3}$ ). The diagram on the left-hand side will contribute to $a_{\mu}$, which is given by [44-46]

$$
\begin{aligned}
a_{\mu}= & \sum_{i} \frac{\lambda_{2 i}^{E} \lambda_{2 i}^{E *} m_{\mu}^{2}}{16 \pi^{2}}\left[\frac{1}{M_{H^{0}}^{2}}\left(\xi_{1}\left(r_{E_{i}}^{H^{0}}\right)-\xi_{2}\left(r_{E_{i}}^{H^{0}}\right)\right)\right. \\
& +\frac{1}{M_{A^{0}}^{2}}\left(\xi_{1}\left(r_{E_{i}}^{A^{0}}-\xi_{2}\left(r_{E_{i}}^{A^{0}}\right)\right)\right]
\end{aligned}
$$

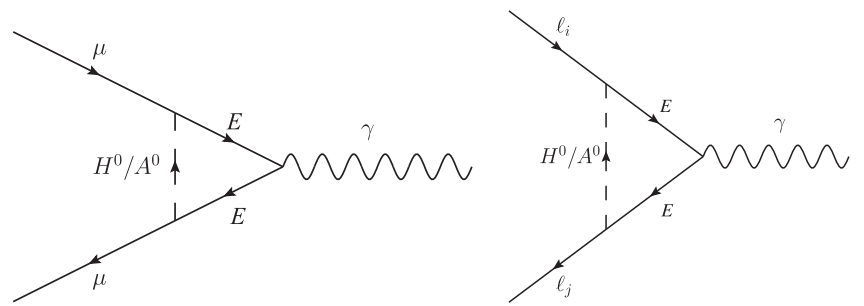

FIG. 2. Feynman diagrams contributing to muon anomalous magnetic moment $a_{\mu}$ (left) and lepton flavor violating decays (right). Here, $E\left(=E_{1} / E_{2} / E_{3}\right)$ is the vectorlike lepton. with $r_{E_{i}}^{X}=m_{E_{i}}^{2} / M_{X}^{2}\left(X=H^{0}\right.$ or $\left.A^{0}\right)$. Here, $m_{E_{i}}$ is the mass of the $i$ th generation vectorlike lepton and $\lambda_{2 i}^{E}$ is the corresponding coupling to muon as expressed in Eq. (10). Note that in the Yukawas of the vectorlike fermions, the first index corresponds to the SM fermion generation while the second index represents that for vectorlike fermions. The functions $\xi_{1}$ and $\xi_{2}$ are given by

$\xi_{1}(r)=\frac{-3+4 r-r^{2}}{2(1-r)^{3}}-\frac{\ln r}{(1-r)^{3}}$

$\xi_{2}(r)=\frac{1}{6(1-r)^{4}}\left[-11+18 r-9 r^{2}+2 r^{3}-6 \ln r\right]$.

The contributions to the decay $\ell_{i} \rightarrow \ell_{j} \gamma$ will be obtained from the rhs diagram of Fig. 2, which is given as $[45,46]$

$$
\begin{aligned}
A_{R}= & \sum_{k} \frac{\lambda_{i k}^{E} \lambda_{j k}^{E}{ }^{*} m_{\ell_{i}}}{16 \pi^{2}}\left[\frac{1}{M_{H^{0}}^{2}}\left(\xi_{1}\left(r_{E_{k}}^{H^{0}}\right)-\xi_{2}\left(r_{E_{k}}^{H^{0}}\right)\right)\right. \\
& \left.+\frac{1}{M_{A^{0}}^{2}}\left(\xi_{1}\left(r_{E_{k}}^{A^{0}}\right)-\xi_{2}\left(r_{E_{k}}^{A^{0}}\right)\right)\right] .
\end{aligned}
$$

In this section we have only shown the analytical expressions of various contributions in $a_{\mu}$ and $\mathcal{B}\left(\ell_{i} \rightarrow \ell_{j} \gamma\right)$, the numerical results are presented in Sec. VI.

\section{NP CONTRIBUTIONS IN $b \rightarrow s$ DECAYS}

$$
\text { A. } b \rightarrow s \ell^{+} \ell^{-} \text {decays }(\ell=\mu, e)
$$

As mentioned earlier, the FCNC transitions such as $b \rightarrow s$ are important probes of flavor physics and are highly sensitive to NP contributions. The effective Hamiltonian for the $b \rightarrow s$ transitions at low energy can be written as $[47,48]$

$$
\begin{aligned}
\mathcal{H}_{\text {eff }}= & -\frac{4 G_{F}}{\sqrt{2}} V_{t b} V_{t s}^{*}\left(\sum_{i=1 \ldots 6} C_{i} \mathcal{O}_{i}\right. \\
& \left.+\sum_{i=7,8,9,10, S, P}\left(C_{i} \mathcal{O}_{i}+C_{i}^{\prime} \mathcal{O}_{i}^{\prime}\right)\right)+ \text { H.c. },
\end{aligned}
$$

where $O_{i}$ and $O_{i}^{\prime}$ 's are the dimension six effective operators which are given as below: 


$$
\begin{array}{rlrl}
\mathcal{O}_{1} & =\left(\bar{s}_{\alpha} c_{\beta}\right)_{V-A}\left(\bar{c}_{\beta} b_{\alpha}\right)_{V-A}, & & \mathcal{O}_{2}=(\bar{s} c)_{V-A}(\bar{c} b)_{V-A}, \\
\mathcal{O}_{3} & =(\bar{s} b)_{V-A} \sum_{q}(\bar{q} q)_{V-A}, & \mathcal{O}_{4} & =\left(\bar{s}_{\alpha} b_{\beta}\right)_{V-A} \sum_{q}\left(\bar{q}_{\beta} q_{\alpha}\right)_{V-A}, \\
\mathcal{O}_{5} & =(\bar{s} b)_{V-A} \sum_{q}(\bar{q} q)_{V+A}, & \mathcal{O}_{6} & =\left(\bar{s}_{\alpha} b_{\beta}\right)_{V-A} \sum_{q}\left(\bar{q}_{\beta} q_{\alpha}\right)_{V+A}, \\
\mathcal{O}_{7} & =\frac{e}{g^{2}} m_{b}\left(\bar{s} \sigma_{\mu \nu} P_{R} b\right) F^{\mu \nu}, & \mathcal{O}_{7}^{\prime} & =\frac{e}{g^{2}} m_{b}\left(\bar{s} \sigma_{\mu \nu} P_{L} b\right) F^{\mu \nu}, \\
\mathcal{O}_{8} & =\frac{1}{g} m_{b}\left(\bar{s} \sigma_{\mu \nu} T^{a} P_{R} b\right) G^{\mu \nu}, & \mathcal{O}_{8}^{\prime} & =\frac{1}{g} m_{b}\left(\bar{s} \sigma_{\mu \nu} T^{a} P_{L} b\right) G^{\mu \nu}, \\
\mathcal{O}_{9} & =\frac{e^{2}}{g^{2}}\left(\bar{s} \gamma_{\mu} P_{L} b\right)\left(\bar{l} \gamma^{\mu} l\right), & \mathcal{O}_{9}^{\prime} & =\frac{e^{2}}{g^{2}}\left(\bar{s} \gamma_{\mu} P_{R} b\right)\left(\bar{l} \gamma^{\mu} l\right), \\
\mathcal{O}_{10} & =\frac{e^{2}}{g^{2}}\left(\bar{s} \gamma_{\mu} P_{L} b\right)\left(\bar{l} \gamma^{\mu} \gamma_{5} l\right), & \mathcal{O}_{10}^{\prime} & =\frac{e^{2}}{g^{2}}\left(\bar{s} \gamma_{\mu} P_{R} b\right)\left(\bar{l} \gamma^{\mu} \gamma_{5} l\right), \\
\mathcal{O}_{S} & =\frac{e^{2}}{16 \pi^{2}}\left(\bar{s} P_{L} b\right)(\bar{l} l), & \mathcal{O}_{S}^{\prime} & =\frac{e^{2}}{16 \pi^{2}}\left(\bar{s} P_{R} b\right)(\bar{l} l), \\
\mathcal{O}_{P} & =\frac{e^{2}}{16 \pi^{2}}\left(\bar{s} P_{L} b\right)\left(\bar{l} \gamma_{5} l\right), & \mathcal{O}_{P}^{\prime}=\frac{e^{2}}{16 \pi^{2}}\left(\bar{s} P_{R} b\right)\left(\bar{l} \gamma_{5} l\right),
\end{array}
$$

where $\alpha$ and $\beta$ denote the color indices and the labels $(V \pm A$ ) refer to $\gamma_{\mu}\left(1 \pm \gamma_{5}\right)$, and $P_{L, R}=\left(\frac{1 \mp \gamma_{5}}{2}\right)$. The operators $\mathcal{O}_{1}$ to $\mathcal{O}_{10}$ appear in the SM effective theory, as well as in specific BSM scenarios, while the rest will appear only in NP models. The Wilson coefficients $\left(C_{i} \mathrm{~s}\right)$ corresponding to the SM effective operators can be found in [49]. The operators relevant for the decay $b \rightarrow s \ell^{+} \ell^{-}$are given by $\mathcal{O}_{9,10}^{(\prime)}$. However, only $\mathcal{O}_{9,10}$ can explain the observed pattern in $R\left(K^{(*)}\right)$ [19]. The expression for the decay rate corresponding to the operator basis given in Eq. (27) are taken from [47].

Another $b \rightarrow s \mu^{+} \mu^{-}$transition that plays a major role in constraining the NP parameter spaces is the rare decay $B_{s} \rightarrow \mu^{+} \mu^{-}$. In the SM, this decay occurs via the penguin and the box diagrams, and is helicity suppressed. In the operator basis mentioned in Eq. (27), only $\mathcal{O}_{10}$ contributes to this process within SM. The corresponding expression for the branching fraction is given by

$$
\begin{aligned}
\mathcal{B}\left(B_{s} \rightarrow l^{+} l^{-}\right)^{\mathrm{SM}}= & \tau_{B_{s}} \frac{G_{F}^{2} \alpha^{2}}{16 \pi^{3}}\left|V_{t b} V_{t s}^{*}\right| m_{B_{s}} m_{\mu}^{2} \\
& \times \sqrt{1-\frac{4 m_{\mu}^{2}}{m_{B_{s}}^{2}}} f_{B_{s}}^{2}\left|C_{10}\right|^{2} .
\end{aligned}
$$

The SM prediction [50] and the measured value [7,51] of the branching fraction for this particular rare decay are respectively given by

$$
\begin{gathered}
\mathcal{B}\left(B_{s} \rightarrow \mu^{+} \mu^{-}\right)^{\mathrm{SM}}=(3.65 \pm 0.23) \times 10^{-9}, \\
\mathcal{B}\left(B_{s} \rightarrow \mu^{+} \mu^{-}\right)^{\mathrm{Expt}}=\left(2.7_{-0.5}^{+0.6}\right) \times 10^{-9} .
\end{gathered}
$$

We note that the measured value and the SM prediction are consistent with each other within the error bars. This, in turn, will be helpful to constrain new physics parameters.

In the BSM framework, there are several dimension six effective operators which may contribute to the process $B_{s} \rightarrow \mu^{+} \mu^{-}$. In the operator basis of Eq. (27), the expression for the branching fraction will then be modified to

$$
\begin{aligned}
\mathcal{B}\left(B_{s}\right. & \left.\rightarrow \mu^{+} \mu^{-}\right)^{\mathrm{BSM}} \\
= & \tau_{B_{s}} f_{B_{s}}^{2} m_{B_{s}}^{3} \frac{G_{F}^{2} \alpha^{2}}{64 \pi^{3}}\left|V_{t b} V_{t s}^{*}\right| \\
& \times \sqrt{1-\frac{4 m_{\mu}^{2}}{m_{B_{s}}^{2}}}\left[\frac{m_{B_{s}}^{2}}{m_{b}^{2}}\left(1-\frac{4 m_{\mu}^{2}}{m_{B_{s}}^{2}}\right)\left|C_{S}-C_{S}^{\prime}\right|^{2}\right. \\
& \left.+\left|\frac{m_{B_{s}}}{m_{b}}\left(C_{P}-C_{P}^{\prime}\right)+2 \frac{m_{\mu}}{m_{B_{s}}}\left(C_{10}-C_{10}^{\prime}\right)\right|^{2}\right] .
\end{aligned}
$$

Here, $C_{S}^{(\prime)}$ and $C_{P}^{(\prime)}$ are the Wilson coefficients associated with the scalar and pseudoscalar operators. It has been shown that these operators are tightly constrained by the data on $\mathcal{B}\left(B_{s} \rightarrow \mu^{+} \mu^{-}\right)$. Therefore, the contributions from the scalar and pseudoscalar operators cannot explain the observed anomalous results in $R\left(K^{(*)}\right)$ [52,53].

In our model, the diagrams that will contribute to the process $b \rightarrow s \ell \ell$ are shown in Fig. 3, where $X / X^{\prime}$ can be either of $H^{0}$ or $A^{0}$. As one can see from Eq. (10), the new couplings $\left(\lambda_{i j}^{E, D}\right)$ carry the generation indices of the SM fields (first index) as well as that of the vectorlike fermions (second index). Therefore, depending on the type of vectorlike fermion in the loop, there will be several 

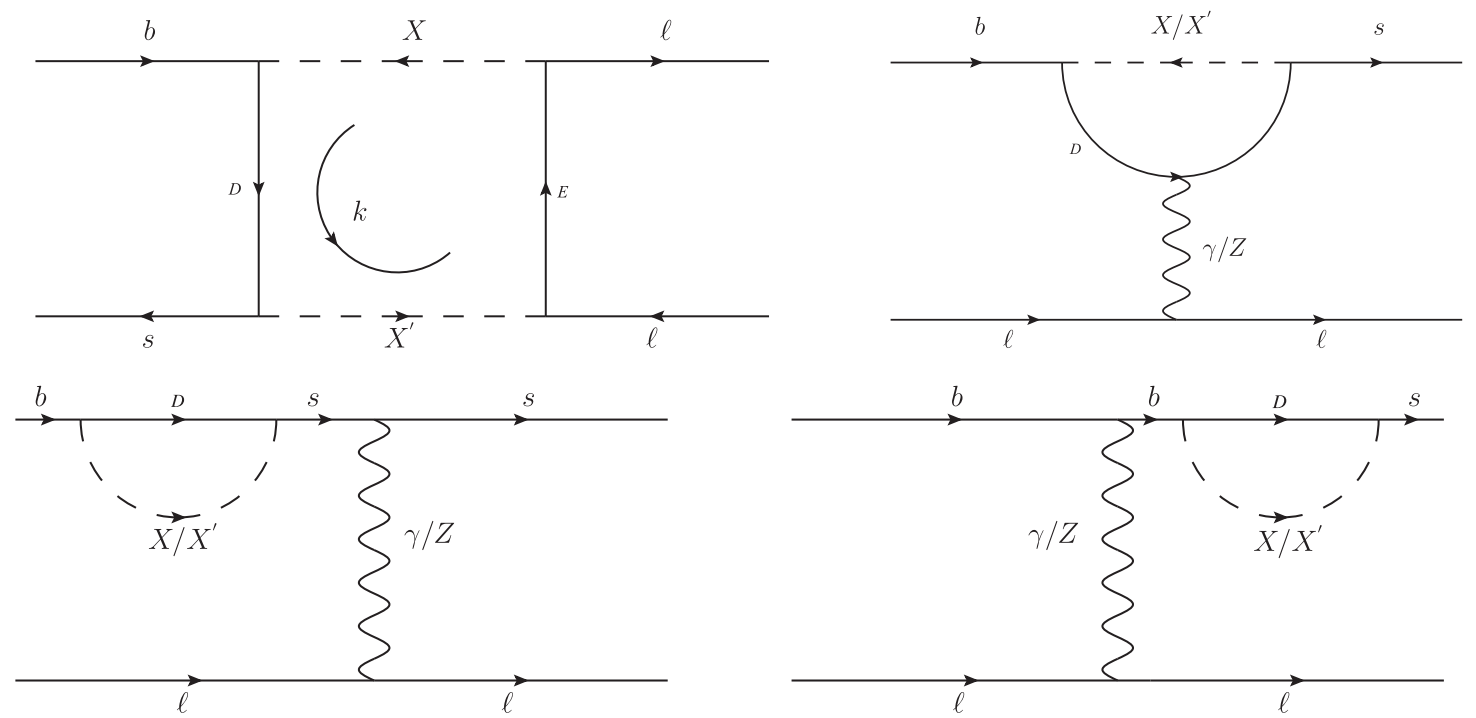

FIG. 3. Feynman diagrams contributing to the $b \rightarrow s \ell \ell$ process. Here $X$ and $X^{\prime}$ can be either of $H^{0}$ or $A^{0}$. The box diagrams with $X=X^{\prime}=H^{0} / A^{0}$ will also contribute to $b \rightarrow$ sle processes.

contributions to the decay amplitude. This will be a function of the new Yukawa couplings and the masses of the new particles. However, for the simplicity of the analysis, we have followed the hierarchy: $\lambda_{i j}^{E, D} \ll \lambda_{i i}^{E, D}$ (i, $\mathrm{j}=1,2$ and 3), i.e., the off diagonal Yukawas are suppressed with respect to the diagonal terms. Also, since one of our goals is to explain the $R\left(K^{(*)}\right)$ anomaly, which requires lepton universality violation, we have further assumed $\lambda_{33}^{E, D} \gg \lambda_{22}^{E, D} \gg \lambda_{11}^{E, D}$. In this simplified picture, the box diagram with $D_{3}$ and $E_{2}$ (in the loop) will have the dominant contribution to the process $b \rightarrow s \mu^{+} \mu^{-}$. Since the dominant contribution to all the observables mentioned above occur via the third generation of down-type vectorlike fermion $D_{3}$ (due to hierarchy in the couplings), we will from now on talk only about the mass of $D_{3}$. In general, the contributions from the penguin diagrams are dominant over that of the box diagrams. However, the penguin diagrams alone cannot explain $R\left(K^{(*)}\right)$ anomaly, as they contribute equally to the decay rates of $B \rightarrow K^{(*)} \mu \mu$ and $B \rightarrow K^{(*)} e e$. Perhaps it is possible to explain the observed data by considering contributions from the new box diagrams alone. In such cases, the interference of the SM Wilson coefficients (WC) with that obtained from the box diagrams will play the leading role in explaining the observed pattern in $R\left(K^{(*)}\right)$ data. If we add the contributions from the penguin diagrams, then there will be interference of the WC obtained from the box and the penguin diagrams. Hence, depending on the size of the individual contributions, the interference of the new box and penguin diagrams could also play an important role in the explanation of the observed data. For completeness, in our analysis we have considered the contributions from all types of diagrams which are shown in Fig. 3.
The most general expression for the box diagram with two different scalars $X$ and $X^{\prime}$ in the loop is given by

$$
i \mathcal{M}_{\mathrm{Box}}=\frac{i \pi^{2} \lambda_{3 i}^{D *} \lambda_{2 i}^{D} \lambda_{2 j}^{E *} \lambda_{2 j}^{E}}{(2 \pi)^{4}} \mathcal{A \mathcal { O } _ { \mathrm { eff } }}
$$

the loop factor is given by [45]

$$
\begin{aligned}
\mathcal{A}= & {\left[\frac{M_{X^{\prime}}^{4}}{\left(M_{X}^{2}-M_{X^{\prime}}^{2}\right)\left(M_{D_{i}}^{2}-M_{X^{\prime}}^{2}\right)\left(M_{E_{j}}^{2}-M_{X^{\prime}}^{2}\right)} \ln \left(\frac{M_{X}^{2}}{M_{X^{\prime}}^{2}}\right)\right.} \\
& +\frac{M_{D_{i}}^{4}}{\left(M_{D_{i}}^{2}-M_{E_{j}}^{2}\right)\left(M_{D_{i}}^{2}-M_{X^{\prime}}^{2}\right)\left(M_{D_{i}}^{2}-M_{X}^{2}\right)} \ln \left(\frac{M_{D_{i}}^{2}}{M_{X}^{2}}\right) \\
& \left.+\frac{M_{E_{j}}^{4}}{\left(M_{E_{j}}^{2}-M_{D_{i}}^{2}\right)\left(M_{E_{j}}^{2}-M_{X^{\prime}}^{2}\right)\left(M_{E_{j}}^{2}-M_{X}^{2}\right)} \ln \left(\frac{M_{E_{j}}^{2}}{M_{X}^{2}}\right)\right] .
\end{aligned}
$$

Also, we have added the contributions of the box diagrams with only one type of scalar $\left(H^{0}, A^{0}\right)$ in the loop. The expression for this can be found from Eq. (33) by taking the limiting case $X \rightarrow X^{\prime}$.

The effective operator is given by

$$
\begin{aligned}
\mathcal{O}_{\text {eff }} & =\left[\bar{b} \gamma_{\mu}\left(1-\gamma_{5}\right) s\right]\left[\bar{l} \gamma^{\mu}\left(1-\gamma_{5}\right) l\right] \\
& =\left[\bar{b} \gamma_{\mu}\left(1-\gamma_{5}\right) s\right]\left[\bar{l} \gamma^{\mu} l\right]-\left[\bar{b} \gamma_{\mu}\left(1-\gamma_{5}\right) s\right]\left[\bar{l} \gamma^{\mu} \gamma_{5} l\right] \\
& =\mathcal{O}_{9}-\mathcal{O}_{10} .
\end{aligned}
$$

For simplicity, from now on we will rewrite the couplings $\lambda_{33}^{D} \equiv \lambda_{b}, \lambda_{23}^{D} \equiv \lambda_{s}$ and $\lambda_{13}^{D} \equiv \lambda_{d}$. On the other hand, we write $\lambda_{11}^{E} \equiv \lambda_{e}, \lambda_{22}^{E} \equiv \lambda_{\mu}$ and $\lambda_{33}^{E} \equiv \lambda_{\tau}$ to simplify our notations. Thus Eq. (32) can be written as 


$$
i \mathcal{M}_{\mathrm{Box}} \sim i\left[C_{9}^{\mathrm{NP}} \mathcal{O}_{9}+C_{10}^{\mathrm{NP}} \mathcal{O}_{10}\right]
$$

where

$$
C_{9}^{\mathrm{NP}}=-C_{10}^{\mathrm{NP}}=-\left(\frac{\lambda_{b} \lambda_{s} \lambda_{\mu}^{2} \mathcal{A}}{32 \pi^{2}}\right),
$$

which has to be normalized with a factor $\mathcal{N}=$ $-\left(\frac{\sqrt{2}}{4 G_{F} V_{t b}^{*} V_{t s}} \times \frac{4 \pi}{\alpha}\right)$ so that the operators are at par with those given in Eq. (27).

The amplitude of the photon exchanged penguin diagrams can be written as

$\mathcal{M}_{\gamma}=\left[\bar{b}\left(A_{L} q^{2} \gamma_{\mu} P_{L}+i A_{R} m_{\tau} \sigma_{\mu \nu} q^{\nu} P_{R}\right) s\right] \frac{e^{2}}{q^{2}}\left[\bar{\ell} \gamma^{\mu} \ell\right]$,

where $q$ is the photon momentum. The dominant contributions will come from $D_{3}$, therefore, the form factors $A_{L}$ and $A_{R}$ are induced by the product $\lambda_{s} \lambda_{b}$ coupling. The contribution to $C_{9}$ will come only from $A_{L}$, whose approximate form is given by

$$
A_{L}=\frac{\lambda_{s} \lambda_{b}^{*}}{32 \pi^{2} M_{X^{(\prime)}}^{2}} \frac{\xi\left(r_{D_{3}}\right)}{3}
$$

with

$$
\begin{aligned}
\xi\left(r_{D_{3}}\right)= & \frac{1}{6\left(1-r_{D_{3}}\right)^{4}}\left[-11+18 r_{D_{3}}-9 r_{D_{3}}^{2}\right. \\
& \left.+2 r_{D_{3}}^{3}-6 \ln r_{D_{3}}\right],
\end{aligned}
$$

and $r_{D_{3}}=M_{D_{3}}^{2} / M_{X^{(\prime)}}^{2}$.

The $Z$-mediated penguin amplitude for the process $b \rightarrow s \ell \ell$ can be written as

$$
\mathcal{M}_{Z}=\left[\bar{b} F_{L} \gamma_{\mu} P_{L} s\right] \frac{1}{M_{Z}^{2}}\left[\bar{\ell} \gamma^{\mu}\left(a_{L}^{\ell} P_{L}+a_{R}^{\ell} P_{R}\right) \ell\right]
$$

where

$$
\begin{aligned}
& a_{L}^{f}=\frac{g}{\cos \theta_{W}}\left(t_{3}^{f}-Q_{f} \sin ^{2} \theta_{W}\right), \\
& a_{R}^{f}=\frac{g}{\cos \theta_{W}}\left(-Q_{f} \sin ^{2} \theta_{W}\right) .
\end{aligned}
$$

From the diagrams of Fig. 3 we obtain

$$
F_{L}=\frac{g}{\cos \theta_{W}} \frac{\lambda_{s} \lambda_{b}^{*}}{32 \pi^{2}}\left[a_{R}^{D_{3}}\left(\frac{1}{2}-2 C\right)+a_{L}^{D_{3}} r_{D_{3}} \xi_{0}\left(r_{D_{3}}\right)+a_{L}^{s} B\right] .
$$

The finite parts of $C, \xi_{0}$ and $B$ are given by

$$
\begin{aligned}
C & =\frac{1}{2} \int_{0}^{1} d x(1-x) \ln \left[x M_{X^{(\prime)}}^{2}+(1-x) M_{D_{3}}^{2}\right], \\
\xi_{0}\left(r_{D}\right) & =\int_{0}^{1} d x \frac{1-x}{x+(1-x) r_{D_{3}}} \\
B & =\frac{1}{2} \int_{0}^{1} d x x \ln \left[x M_{X^{(1)}}^{2}+(1-x) M_{D_{3}}^{2}\right] .
\end{aligned}
$$

The details of the above calculations can be seen from [45]. The $Z$-mediated penguin diagrams will contribute to both $C_{9}$ and $C_{10}$. Therefore, the total contributions to $C_{9}$ and $C_{10}$ can be extracted from

$$
\mathcal{M}=\mathcal{M}_{\mathrm{Box}}+\mathcal{M}_{Z}+\mathcal{M}_{\gamma}
$$

The numerical analysis is done in Sec. VI.

\section{B. $\boldsymbol{B}_{s}-\overline{\boldsymbol{B}}_{s}$ mixing}

The $\Delta F=2$ process $B_{s}-\bar{B}_{s}$ mixing may play a crucial role in constraining the parameters of our model relevant for $b \rightarrow s$ transitions. In this case the important observable is the mass difference $\Delta M_{B_{s}}$, which is defined as

$$
\Delta M_{B_{s}}=2\left|M_{12}^{B_{s}}\right|
$$

In the SM, the dominating contributions to $M_{12}^{B_{s}}$ will come from the dispersive part of the box diagram amplitude with $W$ boson and the top quark in the loop. The mathematical expression for it is given by

$\left.M_{12}^{B_{s}}\right|_{\mathrm{SM}}=\frac{G_{F}^{2}}{12 \pi^{2}} f_{B_{s}}^{2} \hat{B}_{B_{s}} M_{B_{s}} M_{W}^{2}\left(V_{t b}^{*} V_{t s}\right)^{2} \eta_{B} S_{0}\left(\frac{\bar{m}_{t}^{2}}{M_{W}^{2}}\right)$.

$S_{0}$ is the Inami-Lim function

$$
S_{0}(x)=\frac{4 x-11 x^{2}+x^{3}}{4(1-x)^{2}}-\frac{3 x^{3} \log x}{2(1-x)^{3}}
$$

The detail of the SM calculations can be seen from [54].

In the presence of NP there will be additional contributions to $M_{12}^{B_{s}}$. In our model the dominant contributions will come from the box diagram as shown in Fig. 4, which is given by

$$
\left.M_{12}^{B_{s}}\right|_{N P}=\left(\frac{4 \lambda_{b}^{2} \lambda_{s}^{2}}{32 \pi^{2}}\right) f_{B_{s}}^{2} \hat{B}_{B_{s}} M_{B_{s}} S_{N P}
$$

where 


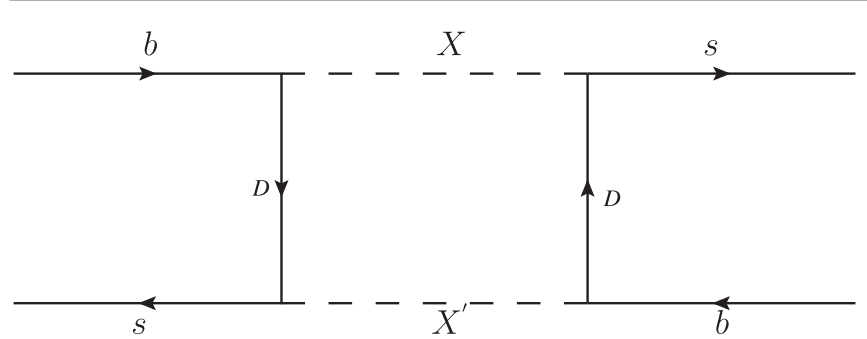

FIG. 4. The Feynman diagram contributing to the $B_{s}$-meson mixing in our model with $X, X^{\prime}$ denoting either $H^{0}$ or $A^{0}$. All other possible symmetric diagrams have also been considered during computation. The dominant contributions will come from $D_{3}$.

$$
\begin{aligned}
S_{N P}= & {\left[\frac{M_{D_{3}}^{2}}{\left(M_{X^{\prime}}^{2}-M_{D_{3}}^{2}\right)\left(M_{D_{3}}^{2}-M_{X}^{2}\right)}\right.} \\
& +\frac{M_{D_{3}}^{4}\left(M_{X}^{2}+M_{X^{\prime}}^{2}\right)-2 M_{D_{3}}^{2} M_{X}^{2} M_{X^{\prime}}^{2}}{\left(M_{X^{\prime}}^{2}-M_{D_{3}}^{2}\right)^{2}\left(M_{D_{3}}^{2}-M_{X}^{2}\right)^{2}} \log \left(\frac{M_{D_{3}}^{2}}{M_{X}^{2}}\right) \\
& \left.-\frac{M_{X^{\prime}}^{4}}{\left(M_{X^{\prime}}^{2}-M_{D_{3}}^{2}\right)^{2}\left(M_{X^{\prime}}^{2}-M_{X}^{2}\right)} \log \left(\frac{M_{X^{\prime}}^{2}}{M_{X}^{2}}\right)\right] .
\end{aligned}
$$

There will be several such diagrams with $H^{0}$ and/or $A^{0}$ in the loop. Our loop factor can be compared with that given in [55].

In the $\mathrm{SM}$, the $B_{s}$ mixing phase is negligibly small, also in our analysis we are assuming real Yukawa couplings. Hence, we can express the mixing amplitude as

$$
\Delta M_{B_{s}}=\left(\Delta M_{B_{s}}\right)_{\mathrm{SM}}+\left(\Delta M_{B_{s}}\right)_{\mathrm{NP}}=\left(\Delta M_{B_{s}}\right)_{\mathrm{SM}}\left(1+\Delta_{\mathrm{Mix}}\right),
$$

with $\Delta_{\mathrm{Mix}}=\frac{\left(\Delta M_{B_{s}}\right)_{\mathrm{NP}}}{\left(\Delta M_{B_{s}}\right)_{\mathrm{SM}}}$. In this ratio, the bag factor and the decay constant will cancel which are the major sources of uncertainties in the SM predictions of the oscillation frequency. $\Delta_{\text {Mix }}$ is sensitive to the NP parameters and using Eq. (50) we can find out the maximum allowed ranges of this observable. Using the latest data on $\Delta M_{B_{s}}$ [7] and the following inputs for decay constant and the bag factor $[56,57]$

$$
\begin{aligned}
& f_{B_{s}}=0.2284 \pm 0.0037 \mathrm{GeV}, \quad \text { and } \\
& B_{B_{s}}=1.327 \pm 0.034
\end{aligned}
$$

we find that $\Delta_{\text {Mix }}$ could be as big as $15 \%$ if we consider the $1 \sigma$ allowed ranges of all the relevant inputs. This could be even $20 \%$ if one uses the projected lattice results as given in Ref. [56]. The $B_{d}-\bar{B}_{d}$ mixing data allows sizable NP contributions $(\approx 30 \%)$ [58]. In our model, the contributions to $B_{d}-\bar{B}_{d}$ mixing will come from the diagram in Fig. 4, with the strange quarks $(s)$ in the external legs replaced by down $(d)$ quarks. The dominant contribution will thus be proportional to $\left(\lambda_{b} \lambda_{d}\right)^{2}$. Therefore, following our assumption of the hierarchical structures of the new Yukawas, the contributions will be highly suppressed. The same argument is also true for $K-\bar{K}$ mixing in which the NP contribution due to our model is proportional to $\left(\lambda_{d} \lambda_{s}\right)^{2}$. Actually, for all practical purposes, we can set $\lambda_{d} \approx 0$. This assumption does not have any impact on our final results since we are mostly interested in the observables which do not have dominant contributions from $\lambda_{d}$.

\section{RESULTS: DM AND FLAVOR}

In this section we discuss the results obtained from the analysis of the DM and flavor sector of our model. We scan the NP parameter space using the constraints from flavor data, relic density and direct detection bounds. In the context of our model, the relevant free parameters are $\lambda_{b}, \lambda_{s}, \lambda_{\mu}, \lambda_{\tau}, M_{H^{0}}, M_{A^{0}}, M_{H^{ \pm}}, M_{E_{3}}, M_{E_{2}}, M_{E_{1}}$, and $M_{D_{1}}=M_{D_{2}}=M_{D_{3}}=M_{D}$.

In order to simplify the analysis, among them we have fixed few of the couplings, such as $\lambda_{s}=0.01$ and $\lambda_{e}=0.001$. Also, we choose $\lambda_{b}<1, \lambda_{\mu}<1.5$ and $\lambda_{\tau} \approx 1.5$. The rest of the free parameters are constrained from the $R\left(K^{(*)}\right), \mathcal{B}\left(B_{s} \rightarrow \mu \mu\right), \Delta a_{\mu}$ and relic data. With these choices of the couplings, we can easily overcome the present constraints on flavor changing $b \rightarrow s$ processes, like $B_{s}-\bar{B}_{s}$ mixing, $\mathcal{B}\left(B \rightarrow X_{s} \gamma\right)$ etc. We will explicitly show this for $B_{s}-\bar{B}_{s}$ mixing. We have checked that in our model within the chosen benchmark points given in Table II, the new physics contribution to the branching fraction $\mathcal{B}\left(B \rightarrow X_{s} \gamma\right)$ will be of order $\mathcal{O}\left(10^{-6}\right)$ which is

TABLE II. Model prediction of different relevant observables corresponding to our chosen benchmark points.

\begin{tabular}{ccccccccccccc}
\hline \hline$B P$ & $M_{D}(\mathrm{GeV})$ & $\lambda_{b}$ & $M_{E_{2}}(\mathrm{GeV})$ & $\lambda_{\mu}$ & $M_{E_{3}}(\mathrm{GeV})$ & $\lambda_{\tau}$ & $\Omega h^{2}$ & $R(K)$ & $R\left(K^{*}\right)$ & $\Delta a_{\mu} \times 10^{10}$ & $\mathcal{B}(\tau \rightarrow \mu \gamma) \times 10^{9}$ & $\mathcal{B}\left(B_{s} \rightarrow \mu \mu\right) \times 10^{9}$ \\
\hline 1 & 500 & 0.9 & 150 & 0.5 & 350 & 1.5 & 0.120 & 0.769 & 0.760 & 3.15 & 5.98 & 1.95 \\
2 & 750 & 0.9 & 250 & 0.7 & 250 & 1.2 & 0.117 & 0.777 & 0.769 & 2.8 & 5.71 & 2.49 \\
3 & 750 & 0.9 & 200 & 0.7 & 350 & 1.5 & 0.122 & 0.758 & 0.749 & 4.01 & 5.39 & 2.55 \\
4 & 850 & 0.9 & 250 & 0.7 & 250 & 1.2 & 0.118 & 0.808 & 0.800 & 2.81 & 5.71 & 2.59 \\
5 & 900 & 0.9 & 350 & 0.8 & 350 & 1.5 & 0.119 & 0.803 & 0.796 & 2.07 & 2.67 & 2.69 \\
6 & 800 & 0.1 & 300 & 1.5 & 350 & 1.5 & 0.121 & 0.908 & 0.903 & 9.5 & 6.03 & 1.89 \\
7 & 800 & 0.1 & 180 & 1.5 & 500 & 1.5 & 0.121 & 0.885 & 0.880 & 4.62 & 5.35 & 1.84 \\
\hline \hline
\end{tabular}




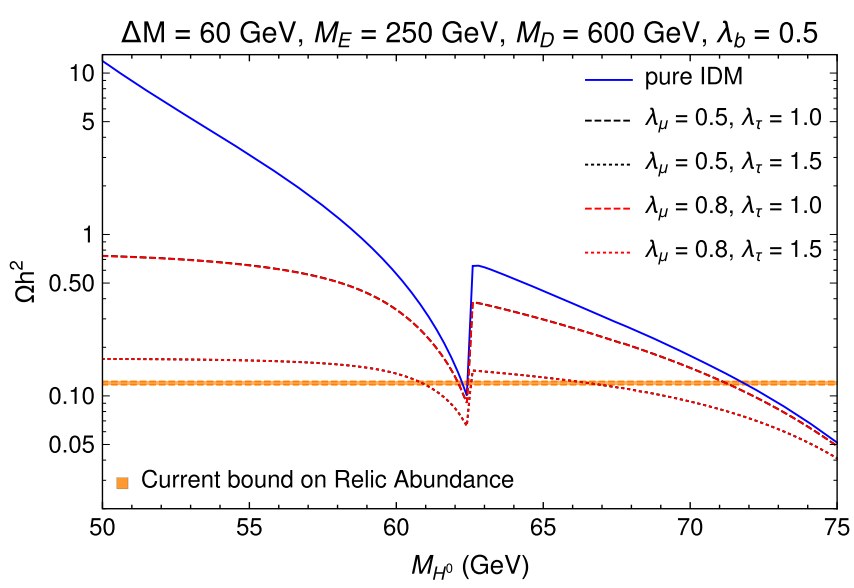

(a)

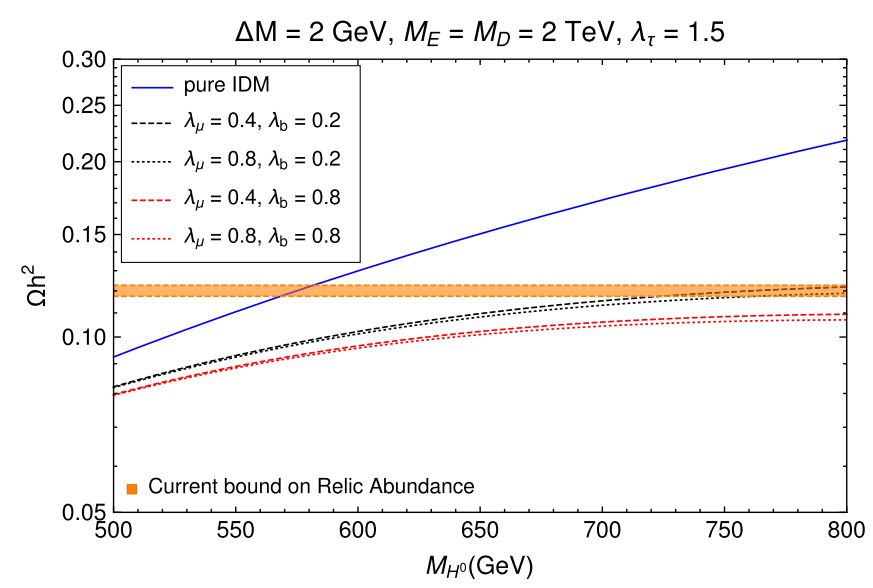

(b)

FIG. 5. Variations of relic abundance with the DM mass $M_{H^{0}}$ for different values of the couplings in the (a) low mass and (b) high mass regions of the DM. The plots show that as we switch on the leptonic and/or quark portal couplings, new annihilation channels open up, lowering the relic abundance for a fixed DM mass. In Fig. 5(a), the black-dashed and red-dashed lines overlap and hence are not visible. This is due to the fact that when $\lambda_{\tau}$ is large, small variations in $\lambda_{\mu}$ do not affect the relic abundance. Similarly, the black and red dotted lines are also overlapped. See the text for more details.

suppressed with respect to the corresponding SM branching fraction by 2 orders of magnitude.

First, we will discuss the effects of different parameters of our model on DM relic abundance. As mentioned earlier, in pure IDM, there exists two distinct regions of DM mass which satisfy the relic abundance criterion. In Fig. 5 we have shown the variations of DM relic abundance with $M_{H^{0}}$ in two different DM mass regions (low and high) for different new couplings and masses of the exotic vectorlike fermions. In Fig. 5(a), we have kept $\Delta M=60 \mathrm{GeV}$ and varied $M_{H^{0}}$ between 50 and $75 \mathrm{GeV}$ (low DM mass region). In this region, with the variation of our new parameters, the allowed values of $M_{H^{0}}$ do not change significantly from that obtained in the pure IDM case. In Fig. 5(b), we have kept $\Delta M=2 \mathrm{GeV}$ and $M_{H^{0}}>500 \mathrm{GeV}$ (high DM mass region). Here, the same mass splitting between different components of the inert scalar doublet namely, $\Delta M=$ $M_{H^{ \pm}}-M_{H^{0}}=M_{A^{0}}-M_{H^{0}}$ is considered. In this region, the deviation from pure IDM scenario is significant. As expected, for the fixed values of the masses of the vectorlike fermions, the new couplings and the associated allowed values of $M_{H^{0}}$ are positively correlated. For the pure IDM scenario, in the low mass region, the allowed values of $M_{H^{0}}$ are not strongly correlated with the choice of $\Delta M$, while in the high mass region the relic abundance is only satisfied when $\Delta M$ is very small, or in other words when the inert scalars are nearly degenerate. Also, in Fig. 5(a), the blackdashed and red-dashed lines overlap with each other. This shows that the DM mostly annihilates to $\tau^{+} \tau^{-}$pairs through vectorlike lepton $E_{3}$ and the annihilation to muon pair is subdominant since the coupling $\lambda_{\tau}$ is much larger than $\lambda_{\mu}$. Hence small changes in $\lambda_{\mu}$ do not affect the relic abundance when $\lambda_{\tau}$ is large for universal vectorlike lepton masses.
For simplicity, in the low DM mass region, we have fixed $M_{H^{0}}$ at $70 \mathrm{GeV}$ for the rest of our analysis. In Fig. 6, we have shown the variations of the relic abundance with the mass splitting $\Delta M$ for different benchmark values of the new couplings and masses. From Figs. 6(a), 6(b) and 6(c) we note that, as the new parameters are switched on, the relic abundance decreases compared to the pure IDM scenario due to the increase in annihilation cross section. Also, the required mass splitting $\Delta M$ will be less in our model compared to that in pure IDM. Since we have assumed $\lambda_{\tau}>\lambda_{\mu}$ the dominant contributions to the relic abundance will come from the annihilation to $\tau^{+} \tau^{-}$, which can be seen from Fig. 6(a) where the variations are almost independent of the choices of $\lambda_{\mu}$. The sensitivity of the relic abundance to the mass splitting in the high mass region is shown in Fig. 6(d). With the increase in $\lambda_{\mu}$, the mass degeneracies are becoming tighter compared to the pure IDM scenario. A similar trend is also expected with the variation of $\lambda_{b}$ as well.

In Sec. IV, we have discussed various diagrams and their contributions to muon $(g-2)$ and LFV decays $\ell_{i} \rightarrow \ell_{j} \gamma$. There will be contributions from penguin diagrams with vectorlike leptons $E_{3}, E_{2}$ or $E_{1}$ in the loop. However, since we are assuming hierarchical structure for the couplings, $\lambda_{21}^{E}<\lambda_{23}^{E} \ll \lambda_{22}^{E}\left(=\lambda_{\mu}\right) \lesssim \lambda_{33}^{E}\left(\equiv \lambda_{\tau}\right)$, the diagrams with $E_{3}$ and $E_{1}$ in the loop will not give significant contributions to $\Delta a_{\mu}$. However for completeness, we consider all those contributions in our analysis. The dominant contribution will come from the penguin diagram with $E_{2}$ in the loop. The variations of $\Delta a_{\mu}$ with the new coupling $\lambda_{\mu}$ for different values of $M_{E_{2}}$ are shown in Fig. 7(a). We note that if we restrict ourselves to the values of $\lambda_{\mu}<1.5$, then it will be 


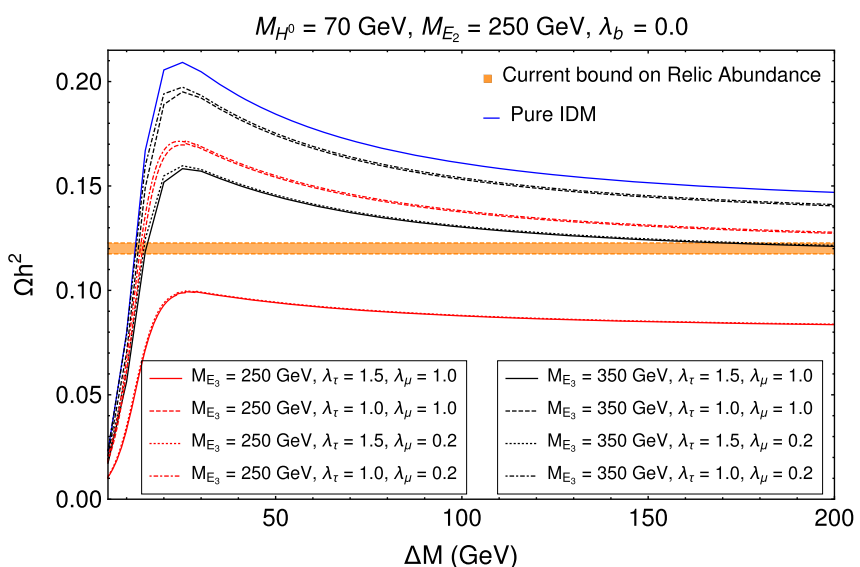

(a)

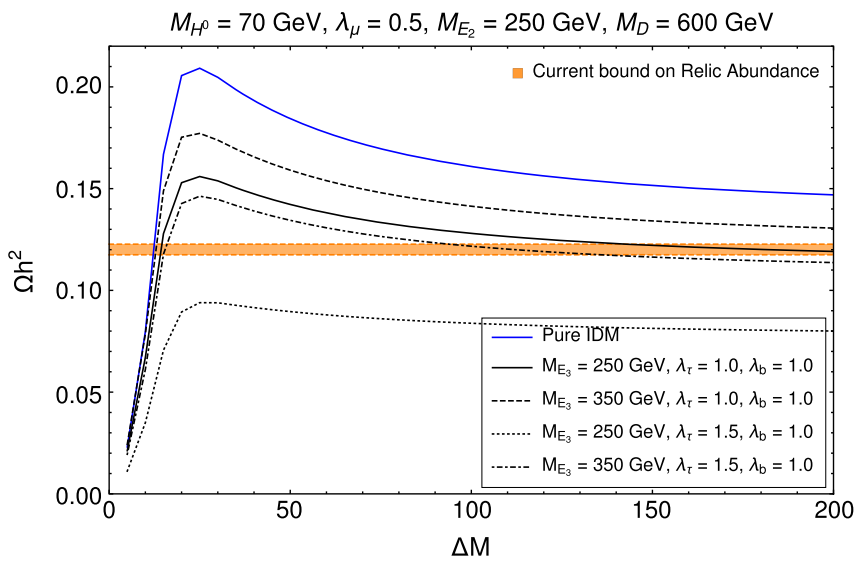

(c)

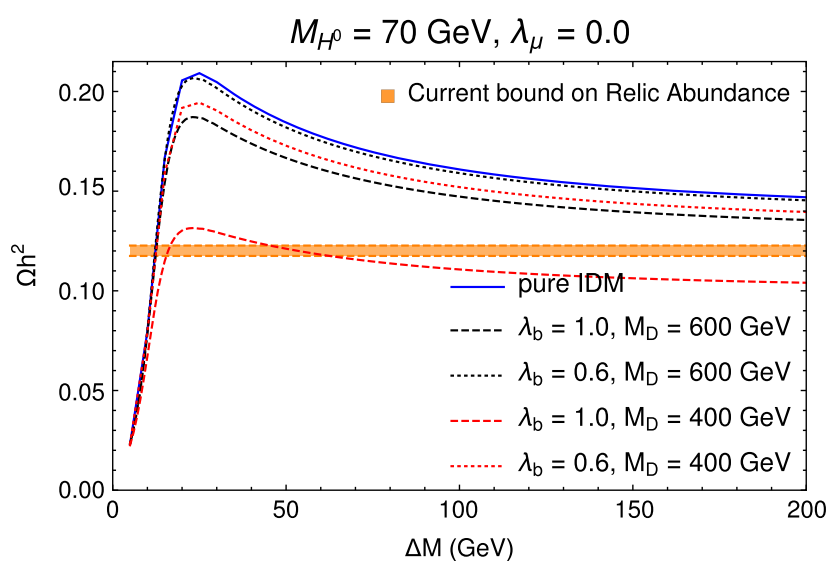

(b)

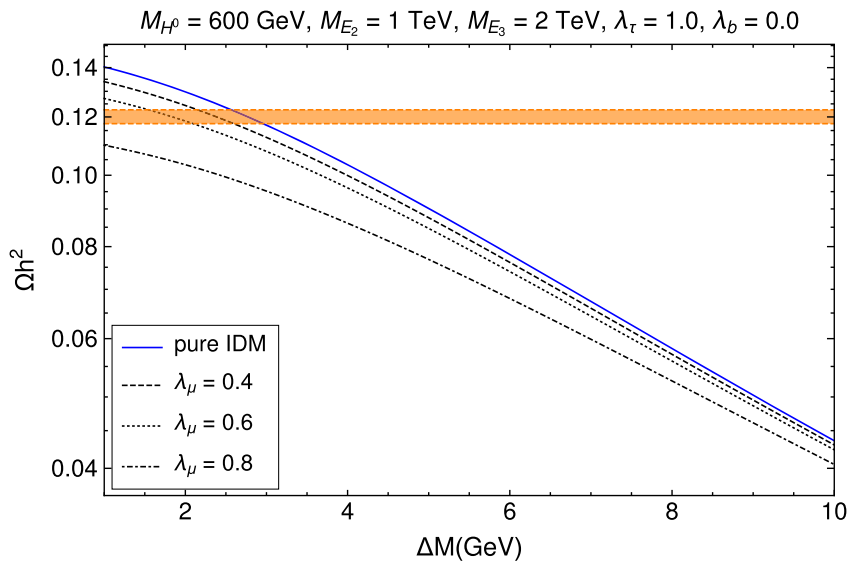

(d)

FIG. 6. The plots in the top panels [(a) and (b)] and the bottom left plot (c) show the variations of relic abundance with the mass splitting $\Delta M=M_{H^{ \pm}}-M_{H^{0}}=M_{A^{0}}-M_{H^{0}}$ for the DM mass $M_{H^{0}}=70 \mathrm{GeV}$. Here, different benchmark points are chosen for the other new parameters. Similar correlations in the high DM mass region $\left(M_{H^{0}}=600 \mathrm{GeV}\right)$ are shown in the bottom right plot (d).

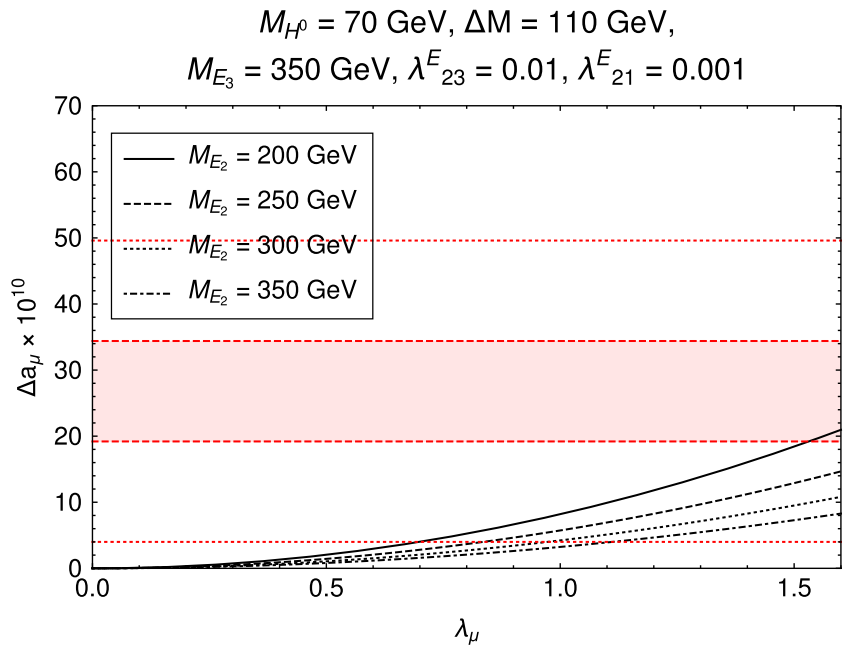

(a)

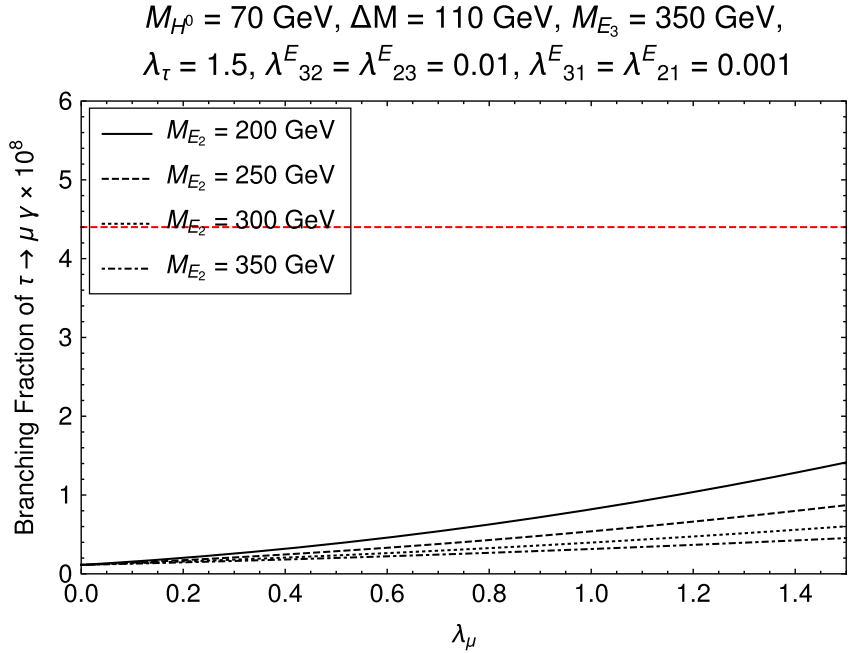

(b)

FIG. 7. The left plot shows the variations of $\Delta a_{\mu}$ [muon $\left.(g-2)\right]$ with the coupling $\lambda_{\mu}$ for different values of $M_{E_{2}}$. In these plots $M_{H^{0}}$ has been taken as $70 \mathrm{GeV}$. The red dashed and dotted lines represent the 1- $\sigma$ and 3- $\sigma$ bands of the $\Delta a_{\mu}$, respectively. The right plot shows that with the same benchmark values of the NP parameters the decay width for $\tau \rightarrow \mu \gamma$ is well below the present experimental limit [7]. 
difficult to explain the excess in muon $(g-2)$ within its $1-\sigma$ range, unless we consider vectorlike lepton mass $\lesssim 200 \mathrm{GeV}$. However, the excess can be successfully explained within its $3-\sigma$ range for values of $\lambda_{\mu} \lesssim 1.5$ and we restrict ourselves to this limit only. Especially, higher masses prefer higher values of the coupling $\lambda_{\mu}$. In the future with more precision measurements, the tension between the predicted and measured values may reduce i.e., the data may become more SM-like. In such situations, the data on $a_{\mu}$ will not put strong constraints on our model parameters. In the opposite situations, one may need to consider values of $\lambda_{\mu} \gtrsim 1.5$. On the other hand, the contribution from all the vectorlike fermions will be relevant for the LFV decays. However, since in our framework the off-diagonal elements are small compared to the diagonal elements, the contribution to the branching fraction will not be significantly large. As an example, we have chosen $\lambda_{32}^{E}=\lambda_{23}^{E} \approx 0.01$. With this choice, the branching fraction $\tau \rightarrow \mu \gamma$ will be much below the current experimental limit, even if we choose $\lambda_{\mu}$ or $\lambda_{\tau}$ roughly $\sim \mathcal{O}(1)$ [Fig. 7(b)]. Here, we have not discussed the LFV $\tau^{-} \rightarrow \mu^{-} \mu^{+} \mu^{-}$decay. In our model, the leading diagram for this decay is the same as $\tau \rightarrow \mu \gamma$, with a virtual photon converting into a muon pair. ${ }^{1}$ It is expected that for the same set of NP parameters the branching fraction $\mathcal{B}\left(\tau \rightarrow \mu^{-} \mu^{+} \mu^{-}\right)$will be small compared to $\mathcal{B}(\tau \rightarrow \mu \gamma)$; as an example see [45]. Therefore, our NP parameters will be safe with respect to the present limit $\mathcal{B}(\tau \rightarrow \mu \mu \mu)\left(\approx \mathcal{O}\left(10^{-8}\right)\right)$ [7]. The LFV decays and $\Delta a_{\mu}$ are insensitive to the coupling $\lambda_{b}$. However, observables like $R\left(K^{(*)}\right)$ and DM relic abundance are sensitive to all the relevant couplings and masses of the model.

In the case of $B_{s}-\bar{B}_{s}$ mixing, in Fig. 8 we have shown the variations of $\Delta_{\text {Mix }}$ with $\lambda_{b}$ for different values of the masses of $M_{D_{3}}$. The black dashed line indicates the $15 \%$ allowed range in $\Delta_{\text {Mix }}$, the solid lines represent our model predictions for different values of the vectorlike quark mass. The other relevant parameters are fixed as before. We note that for $M_{D_{3}} \geq 500 \mathrm{GeV}$, the allowed value of $\lambda_{b}$ could be as big as 1 . However, for lower values of $M_{D_{3}}$ higher values of $\lambda_{b}$ will be disfavored; as an example we can see that for $M_{D_{3}}=300 \mathrm{GeV}, \lambda_{b} \gtrsim 0.7$ will not be allowed. Here, we would like to point out that the major uncertainties in the theory prediction are associated with the decay constant. Therefore, if we consider the errors within their $1 \sigma$ C.L. ranges, then that will give us a conservative estimate of the allowed NP. We would like to stress that data will still allow a NP contribution up to $30 \%-40 \%$ at the $3 \sigma$ C.L. [58].

\footnotetext{
${ }^{1}$ There will be one additional box diagram, the contribution of which will be suppressed compared to that of the penguin diagram.
}

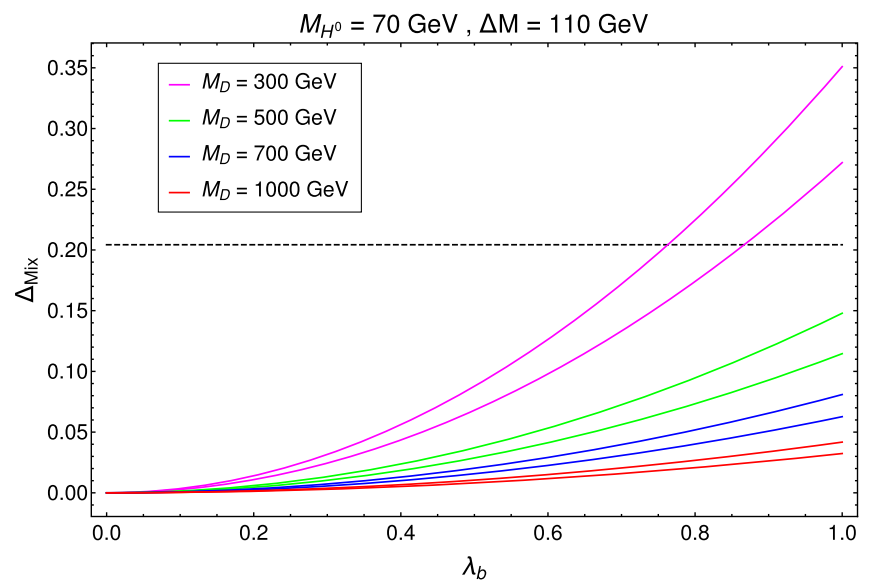

FIG. 8. Variation of $\Delta_{\text {Mix }}$ with $\lambda_{b}$ for four different values of the vectorlike quark mass $M_{D}\left(=M_{D_{3}}\right)$, since the dominant contribution will come from $D=D_{3}$. The black dashed line indicates the maximum allowed value of $\Delta_{\text {Mix }}$ if we take all the inputs in Eq. (50) within their respective $1 \sigma$ confidence interval.

As mentioned earlier, the branching fraction for the rare decay $B_{s} \rightarrow \mu^{+} \mu^{-}$is consistent with its SM prediction within $1 \sigma$ confidence level. Therefore, the data on $\mathcal{B}\left(B_{s} \rightarrow \mu^{+} \mu^{-}\right)$is expected to put tighter constraints on the parameters of any NP model in the decay $b \rightarrow s \mu \mu$. More importantly if we see the current data, the measured value is below that of SM prediction and our model has the potential to accommodate it. Although we are not considering it seriously, we have to wait for more precise data and lattice inputs to conclude it further, but at the moment one cannot rule out this possibility. The main source of error in $B_{s} \rightarrow \mu \mu$ is the decay constant $f_{B_{s}}$ whose different lattice predictions have different errors (for details see Ref. [57]). Therefore, in order to be conservative, the errors in the measured value have been taken in their $2 \sigma$ confidence level allowed ranges to constrain the NP parameters.

From a phenomenology point of view according to the low and high DM mass regions, we divide our analysis into two parts: in one part, we choose $M_{H^{0}}=70 \mathrm{GeV}$ (low DM mass), and in the other we have considered $M_{H^{0}}=600 \mathrm{GeV}$ (high DM mass). These will be discussed in the following sections.

\section{A. Low mass DM}

In this section we show our main results for a light dark matter of mass $70 \mathrm{GeV}$ and mass splitting $\Delta M=110 \mathrm{GeV}$. Keeping in mind all the relevant correlations between the different parameters shown above, we do a multiparameter scan to find out the common parameter space that satisfies all relevant flavor constraints i.e., $R\left(K^{(*)}\right), \mathcal{B}\left(B_{s} \rightarrow \mu^{+} \mu^{-}\right)$, muon magnetic moment anomaly in $\Delta a_{\mu}$ as well as the correct relic abundance and direct detection bounds of dark matter as shown in Fig. 9. To generate these plots we assumed, for simplicity, that all generations of the 


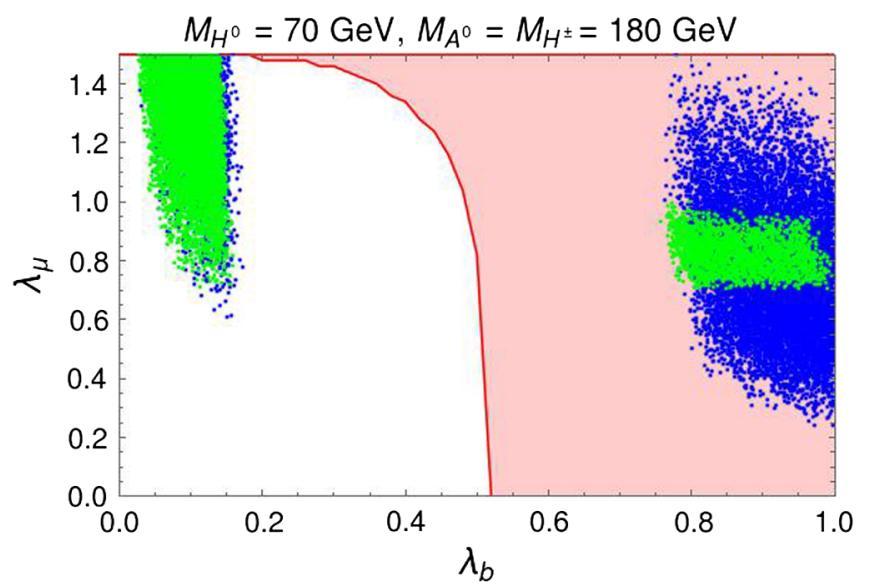

(a)

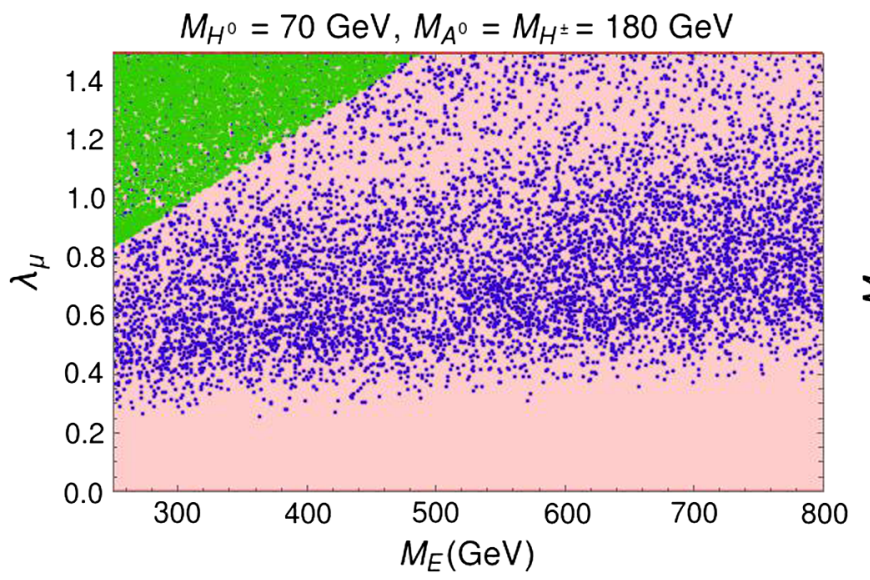

(c)

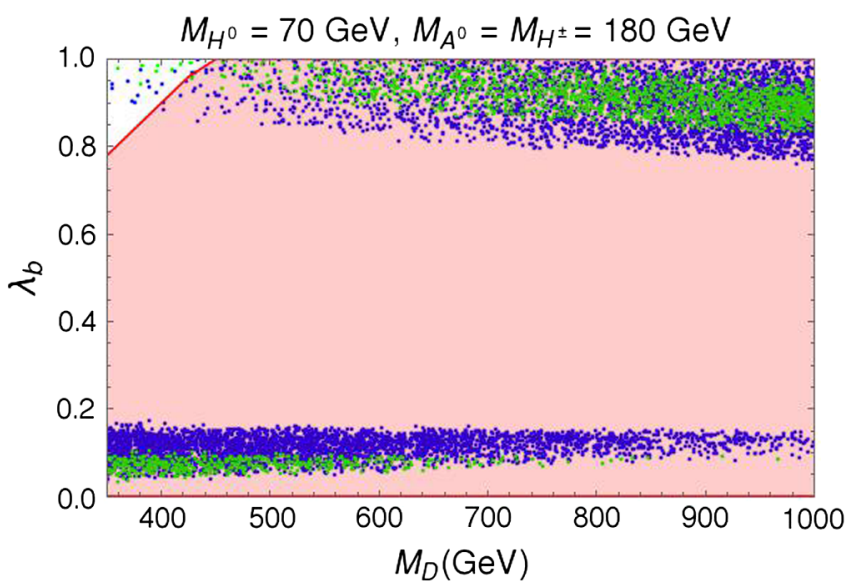

(b)

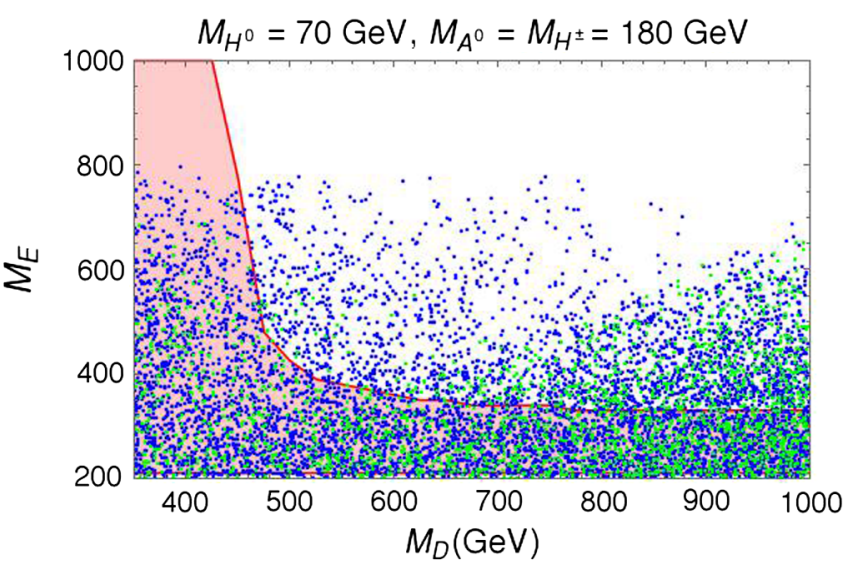

(d)

FIG. 9. Correlation between different NP parameters for a low mass DM at $M_{H^{0}}=70 \mathrm{GeV}$. The blue points satisfy all relevant flavor constraints within their $2 \sigma$ confidence intervals except muon $(g-2)$ anomaly. When we further apply the muon anomalous magnetic moment bounds then the allowed region shrinks as depicted by the green points (see text). The red region satisfies the relic and direct search constraints when we consider degenerate vectorlike fermion masses.

vectorlike leptons have the same mass i.e., $M_{E_{1}}=M_{E_{2}}=$ $M_{E_{3}}=M_{E}$. More regions on the parameter spaces of $\lambda_{\mu}$ and $M_{E_{2}}$ will be allowed if we relax this mass degeneracy. We allow both $M_{E}$ and $M_{D}$ to vary between 200 and $1000 \mathrm{GeV}$ instead of keeping them fixed. In these plots $\lambda_{\tau}$ has been varied between 1.0-1.5, while we have kept $\lambda_{\mu}<1.5$ and $\lambda_{b}<1$. As mentioned earlier, the major constraints on the new parameters are mainly coming from the flavor data, in particular from $\mathcal{B}\left(B_{s} \rightarrow \mu \mu\right)$. The blue scattered points satisfy the data on $R(K), R\left(K^{*}\right)$ and $\mathcal{B}\left(B_{s} \rightarrow \mu \mu\right)$ in their respective $2 \sigma$ confidence intervals which shrink to the green points when we also consider $\Delta a_{\mu}$ as a constraint. Here, we have considered the excess in $\Delta a_{\mu}$ within its 3- $\sigma$ range. The red regions represent the bound on the NP parameters from the relic density and direct detection cross section of DM. An interesting feature here is the presence of two distinct regions in the parameter spaces of $\lambda_{\mu}, \lambda_{b}$ and $M_{D}$ [Figs. 9(a) and 9(b)]. These two regions correspond to high and low values of $\lambda_{b}$, respectively. In both of the allowed regions, $\lambda_{\mu}$ can take moderate values $>0.5$. However, its magnitude cannot be very high (»1) when $\lambda_{b} \gtrsim 0.7$. On the other hand when $\lambda_{b}$ is small, the common parameter spaces are obtained in regions where $\lambda_{\mu} \approx 1.5$, which could be relaxed and the values $\lambda_{\mu} \lesssim 1.5$ will be allowed if we lift the mass degeneracies of the vectorlike leptons, e.g., see Fig. 10(a). ${ }^{2}$ Hence, for completeness we have analyzed both of these regions in the collider searches as will be discussed later. Also, we note that $\lambda_{\mu}>0.4$ for $M_{E} \lesssim 800 \mathrm{GeV}$ and $\lambda_{b}>0.7$ in the whole

\footnotetext{
${ }^{2}$ If we lift the mass degeneracies in the vectorlike leptons, then for higher masses of $M_{E_{3}}$ the DM will mostly annihilate to $\tau^{+} \tau^{-}$ and this channel will contribute maximally to the relic abundance. Hence, there will not be any strong constraints on the parameter spaces of $M_{E_{2}}$ and $\lambda_{\mu}$ from relic abundance which can also be understood from the observations made in Figs. 6(a) and 6(c).
} 


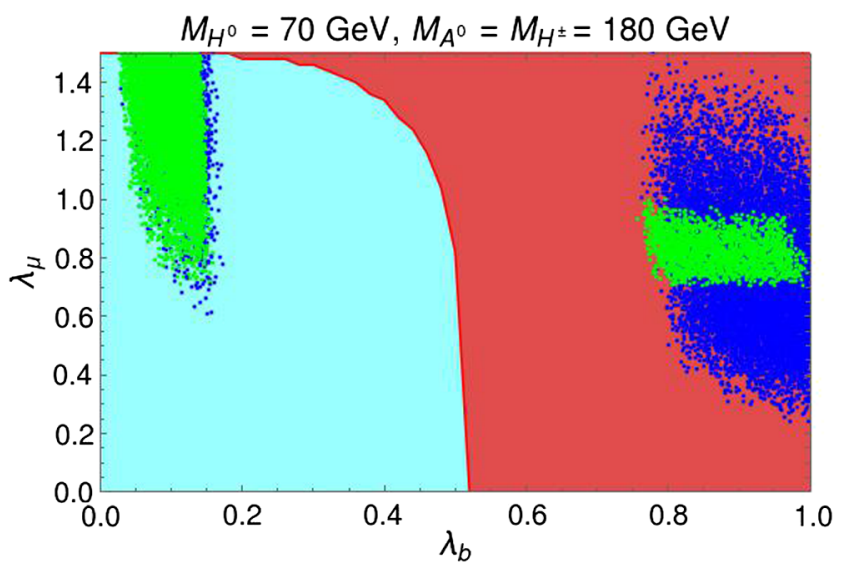

(a)

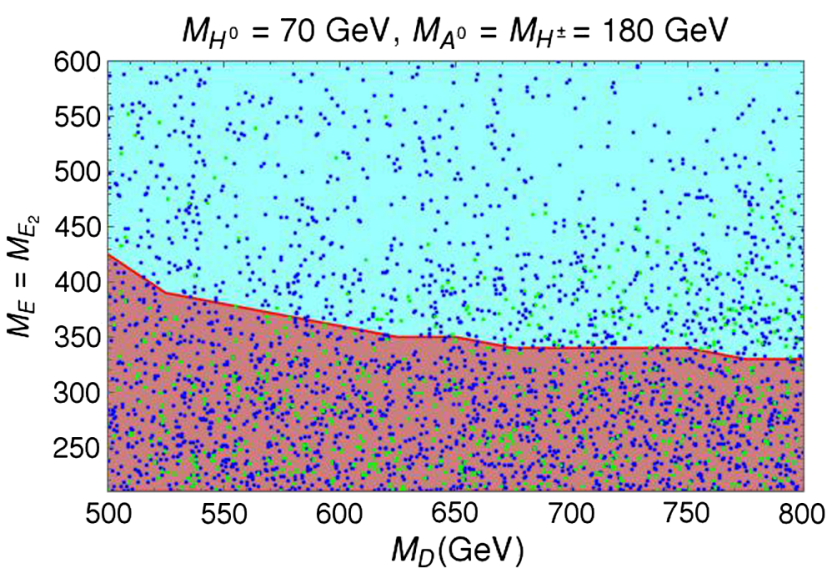

(b)

FIG. 10. Similar plots as given in Figs. 9(a) and 9(d). In addition, we have considered the case $M_{E_{2}} \neq M_{E_{3}}$ which is represented by the cyan region. This region satisfies the relic and direct detection constraints when we assume the nondegenerate vectorlike lepton masses.

range of $M_{D}$ are allowed by all data. Note that muon $(g-2)$ is not sensitive to $\lambda_{b}$ or $M_{D}$. Also, we see a nice correlation between $M_{E}$ and $M_{D}$, for the higher values of $M_{D}$ $(\geq 500 \mathrm{GeV})$ the relic density prefers $M_{E} \leq 400 \mathrm{GeV}$. We have checked that this constraint can be relaxed if we assume nondegenerate vectorlike fermion masses for all the generations, e.g., see Fig. 10(b).

Following the above discussions, from the allowed parameter spaces we have chosen seven benchmark points (BPs), listed in Table. II, for collider analysis elaborated in Sec. VII. Also, we have checked that the values of the Wilson coefficients $C_{9}^{N P}$ and $C_{10}^{N P}$ in all these benchmark scenarios are consistent with those obtained from the global fit [59], in particular the scenario with $C_{9}^{N P}=-C_{10}^{N P}$. We have focused on both the allowed regions of couplings as shown in Fig. 9(a), so that we can phenomenologically distinguish them from each other. BP1 to BP5 have the characteristics of high $\lambda_{b}$ and intermediate $\lambda_{\mu}$ while a low $\lambda_{b}$ and high $\lambda_{\mu}$ characterize BP6 and BP7. Here we would like to mention that there is an existing lower limit on pair-produced charged heavy vectorlike leptons from LEP [60]: $m_{L^{ \pm}} \gtrsim 101.2 \mathrm{GeV}$. Our benchmark points satisfy this limit.

In pure IDM, the electroweak precision observables (EWPO) like $S$ and $T$, play an important role in constraining the mass splitting $\Delta M$ between the inert scalars [30]. We have already taken care of this constraint while scanning the new parameter spaces. Our model contains singlet vector fermions which do not mix. Hence, there will not be any additional significant contributions in $S, T$ and $U$ parameters, although there will be diagrams that contribute to $Z \rightarrow \mu \bar{\mu}$ and $Z \rightarrow b \bar{b}$ decays at one-loop level. However, we have checked that within our chosen model parameters those contributions are highly suppressed. Therefore, the EWPO will not put any stringent constraint on our model parameters.
Before moving on to the collider analysis, we show, for illustrative purpose, the variation of spin-independent direct search cross section (per nucleon) with the DM mass in Fig. 11 for some of the chosen BPs in Table II. For a comparison, the similar correlation for pure IDM is presented in the same figure. The solid red line is the exclusion limit from recent XENON-1T data [32]. The black dots on each black line refer to the particular point corresponding to a fixed $M_{H^{0}}$, satisfying constraints from relic density, direct search (as they lie below the experimental exclusion limit) and flavor bounds (which we have discussed in Secs. IV and VA). As pointed out earlier, and can now be seen from these plots, the presence of exotic quarks increases the direct detection rates compared to the

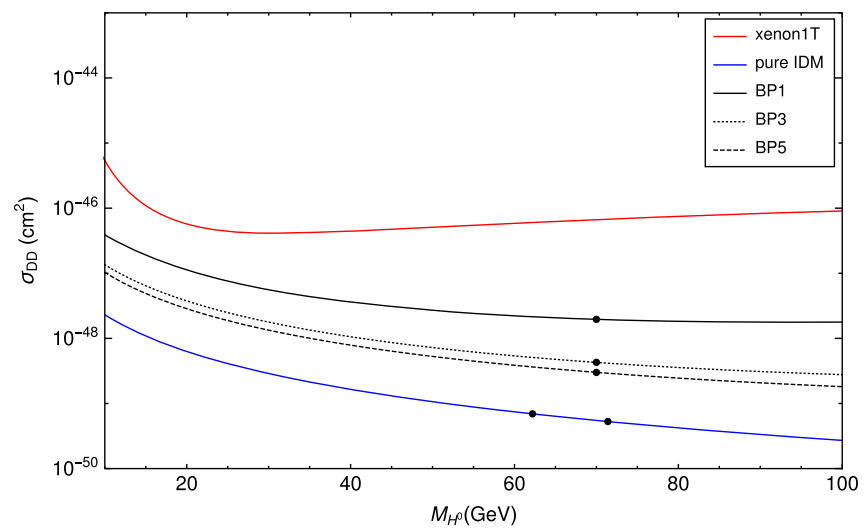

FIG. 11. The plot shows the variation direct detection cross section with DM mass in the direct search plane. The black lines correspond to different BPs, where the DM mass has been varied, while the dots correspond to specific choices of the DM mass (see text for details), the red line is the exclusion limit from recent XENON-1T data and the blue line is the direct search limit from the pure IDM case. 
pure IDM keeping it more promising for observing at ongoing direct search experiments.

\section{B. High mass DM}

We analyze the high DM mass region of the IDM in the context of our extended framework. As discussed earlier, we need to consider degenerate masses for the IDM scalars (as we need to resort on coannihilation channels in order to satisfy relic density) and also tune $\lambda_{L}\left(=\lambda_{3}+\lambda_{4}+\lambda_{5}\right)$, which involves the DM-Higgs interaction, to an appropriate value. So the masses of vectorlike leptons $\left(E_{i}\right)$ and the vectorlike bottom partner $(D)$ will have to be greater than the masses of $A^{0}$ or $H^{ \pm}$to maintain the stability of the DM. We consider a mass splitting of $2 \mathrm{GeV}$ between the inert scalars and set $\lambda_{L}$ to 0.0001 .

The parameter spaces which are allowed by flavor data are shown in Figs. 12(a) and 12(b). We have kept the value of the DM mass fixed at $M_{H^{0}}=600 \mathrm{GeV}$. It is interesting to note that, in this case, we will be able to explain the

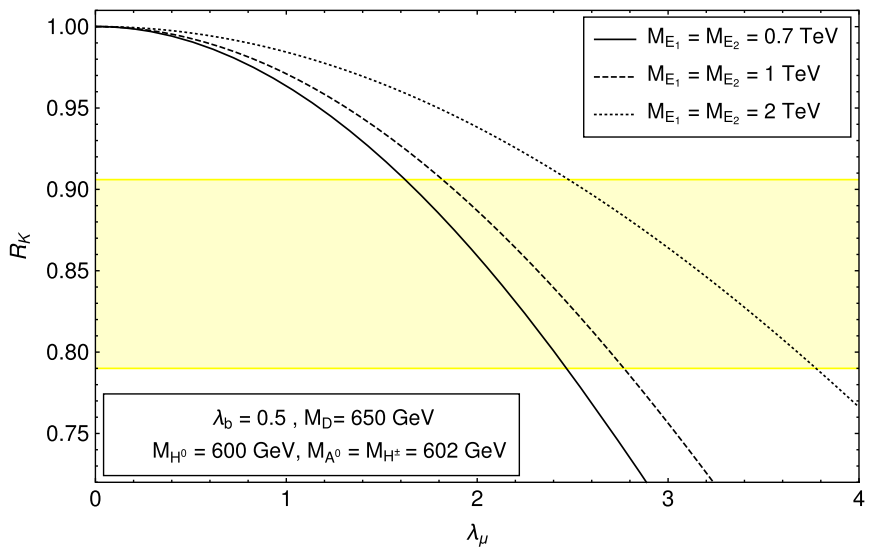

(a)
$R\left(K^{(*)}\right)$ anomaly only for higher values of $\lambda_{\mu}(\approx 3$ or 4$)$. However, for the same masses, such high values of $\lambda_{\mu}$ will not allow us to achieve the right relic abundance [see Fig. 12(c)]. Hence, it is not possible to obtain a common parameter space that satisfies both relic abundance and flavor constraints simultaneously. When we add new interactions, new annihilation channels open up and they make the DM underabundant. So in order to make the effects of NP minimal, we require the couplings to be small but masses to be large [Fig. 5(b)]. However, if we also want to explain the flavor anomalies for such high values of the vectorlike fermions masses, we need very high values of couplings as well $\left(\lambda_{\mu} \gtrsim 3\right)$. So it is impossible to achieve a solution in this region of DM mass and hence we discard further investigations for this case.

\section{COLLIDER PHENOMENOLOGY}

Our goal is to investigate the implications of our model on collider searches at the LHC. As mentioned earlier,

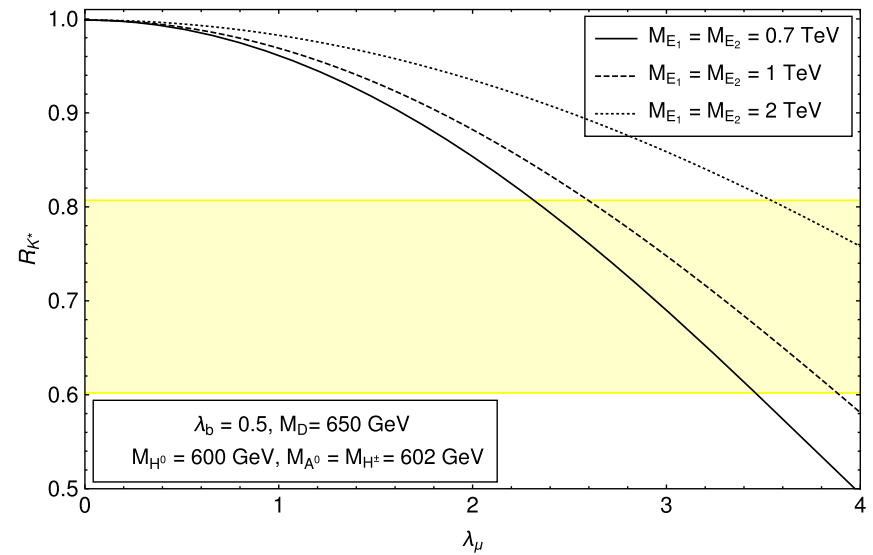

(b)

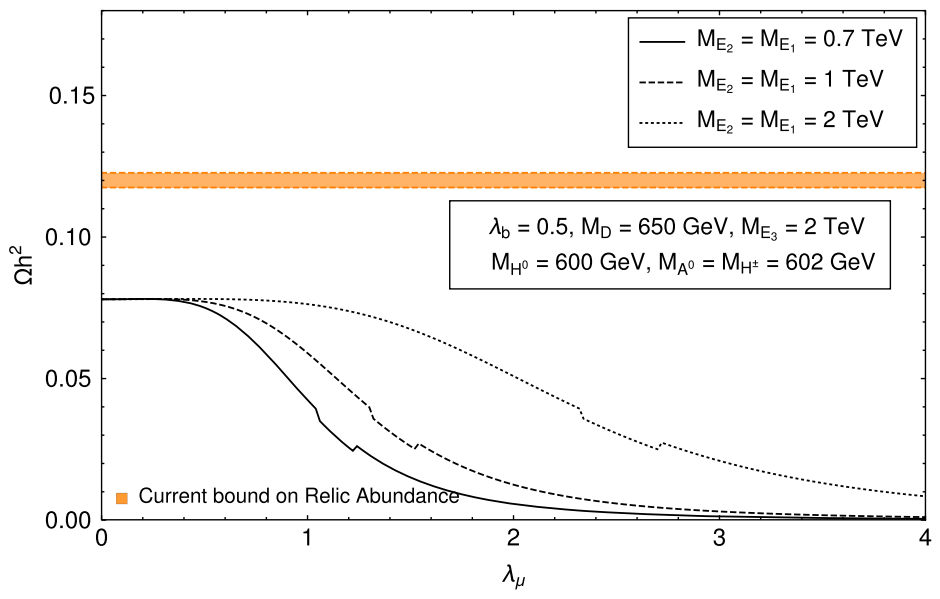

(c)

FIG. 12. Top left: (a) Variation of $R(K)$ with $\lambda_{\mu}$ for fixed values of DM mass and $\lambda_{b}$, for three different values of $M_{E_{2}}$. The yellow band shows the $1 \sigma$ experimental range of $R(K)$. Top right: (b) Same for $1 \sigma$ experimental range of $R\left(K^{(*)}\right)$. Middle: (c) Variation of relic abundance with $\lambda_{\mu}$ for the same chosen parameters as in (a) and (b); the orange band shows the Planck-observed relic density bound. 

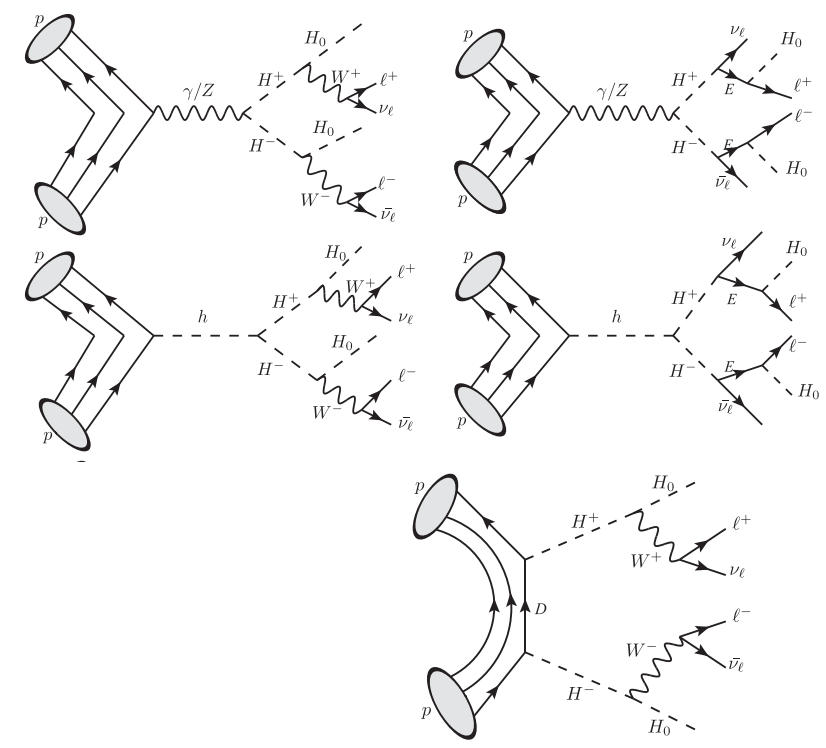
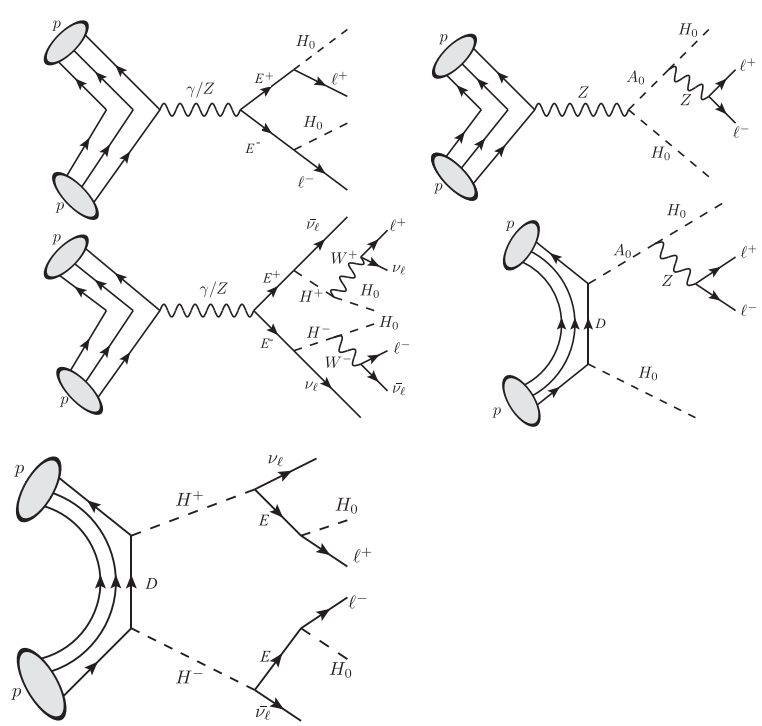

FIG. 13. Feynman diagrams for the production of the $\left(\ell^{+} \ell^{-}+\mathbb{E}_{T}\right)$ final state at a hadron collider like LHC.
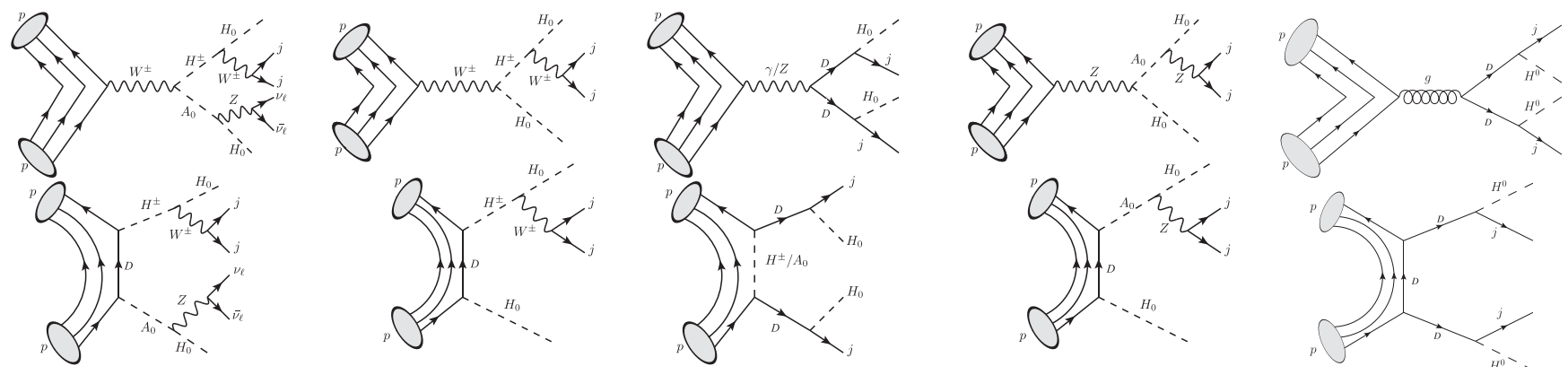

FIG. 14. Feynman diagrams for channels contributing to the $j j+\mathbb{E}_{T}$ final state. The gluon fusion diagram has also been considered in this case.

we have expanded the contact interaction of the DM with the SM and included the vectorlike fermions (mediators) as propagating d.o.f. of the theory. Also, it is clear from the above discussions that the mediators have decay channels to the SM fermions. In this section, we will analyze the prospects for detecting our model at the LHC through various channels. Due to the presence of the exotic vectorlike leptons and quarks, the model gives rise to several tantalizing collider signatures. Here, we have discussed a few of them:

(i) opposite sign dilepton (OSD) with missing energy $\left(\ell^{+} \ell^{-}+\mathbb{E}_{T}\right)^{3}$

(ii) dijet with missing energy $\left(j j+\mathbb{E}_{T}\right)$, there are cases where the b-jets have been tagged separately;

(iii) dilepton with dijet and missing energy $\left(\ell^{+} \ell^{-}+j j+\mathbb{E}_{T}\right)$.

All of these three final states could be tested at the LHC. The corresponding Feynman diagrams for the final states

\footnotetext{
${ }^{3}$ This is also dubbed as Hadronically quiet dilepton with missing energy.
}

$\left(\ell^{+} \ell^{-}+\mathbb{E}_{T}\right),\left(j j+\mathbb{E}_{T}\right)$ and $\left(\ell^{+} \ell^{-}+j j+\mathbb{E}_{T}\right)$ are given in Figs. 13, 14 and 15, respectively. In all of these diagrams, the proton $(p)$ is considered a multiparticle composed of both quarks and gluons. Hence, all possible initial states with quarks and gluons have been taken into account. We note that among all of the diagrams, some will appear only in pure IDM case. Therefore, it is important to test whether it is possible to discriminate the signatures of our model from that of pure IDM at the LHC.

As mentioned earlier, from the common parameter space satisfying flavor constraints, relic density of DM and direct detection bound, we have chosen our benchmark points (BP), which are given in Table II. All other parameters are fixed at the values: $M_{H^{0}}=70 \mathrm{GeV}, \Delta M=110 \mathrm{GeV}$, $m_{h}=125 \mathrm{GeV}, \quad \lambda_{L}=0.0001, \quad \lambda_{2}=0.1, \quad \lambda_{\mathrm{d}}=0.001$, $\lambda_{\mathrm{s}}=0.01, \lambda_{\mathrm{e}}=0.001$.

\section{A. Object reconstruction and simulation strategy}

We implemented the model in FeynRule [61]. The parton level events are generated in MadGraph [62], which are 

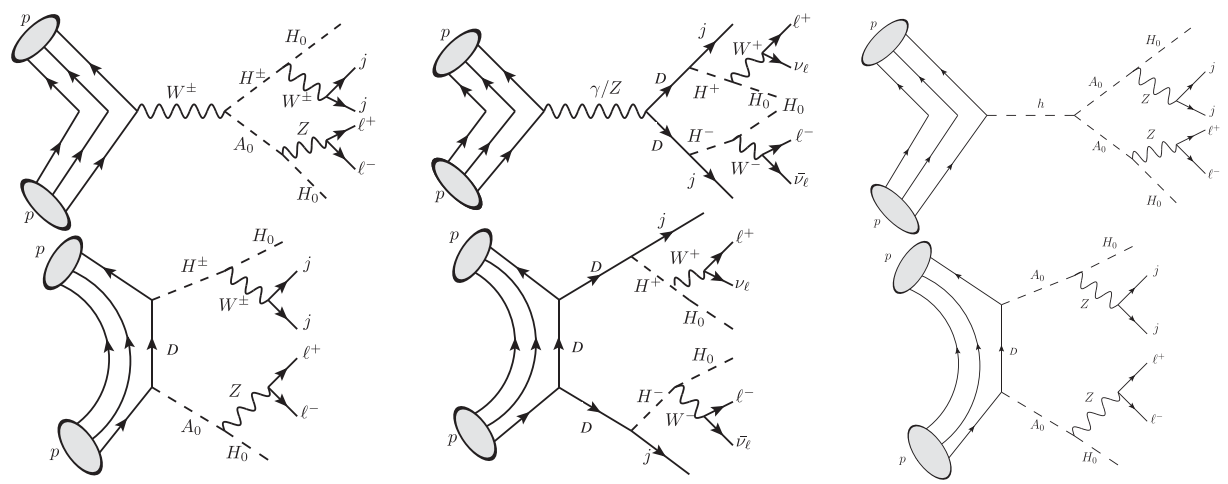

FIG. 15. Feynman diagrams for channels contributing to the $\ell^{+} \ell^{-}+j j+\mathscr{E}_{T}$ final state.

further showered through PYTHIA [63]. All the events are generated at $\sqrt{s}=14 \mathrm{TeV}$ using CTEQ6L [64] as the parton distribution function. All the leptons and jets are reconstructed in order to mimic the LHC environment using the following criteria:

(i) Lepton $(l=e, \mu)$ : Leptons are identified with a minimum transverse momentum $p_{T}>20 \mathrm{GeV}$ and pseudorapidity $|\eta|<2.5$ such that they are in the central part of the detector. Two leptons are distinguished as isolated objects if their mutual distance in the $\eta-\phi$ plane is $\Delta R=\sqrt{(\Delta \eta)^{2}+(\Delta \phi)^{2}} \geq 0.2$, while that separation between an isolated lepton and a jet is given by $\Delta R \geq 0.4$.

(ii) Jets $(j)$ : The cone jet algorithm PYCELL has been used to build jets inside PYTHIA. All of the partons within $\Delta R=0.4$ from the jet initiator cell are included to form the jets. We require $p_{T}>$ $20 \mathrm{GeV}$ for a clustered object to be considered as a jet. Jets are isolated from unclustered objects for $\Delta R>0.4$.

(iii) Unclustered objects: All the final state objects which are neither clustered to form jets nor identified as isolated leptons belong to this category. All particles with $0.5<p_{T}<20 \mathrm{GeV}$ and $|\eta|<5$, are considered as unclustered.

(iv) Missing energy $\left(\mathbb{E}_{T}\right)$ : The transverse momentum of all the missing particles (those are not registered in the detector) can be estimated from momentum imbalance of the visible particles in the transverse direction. Thus, missing energy (MET) is defined as

$$
\boldsymbol{E}_{T}=-\sqrt{\left(\sum_{\ell, j} p_{x}\right)^{2}+\left(\sum_{\ell, j} p_{y}\right)^{2}}
$$

where the sum runs over all visible objects that include the leptons and jets, and the unclustered components.

(v) $H_{T}$ : We have used another observable for collider searches which is the scalar sum of all isolated lepton/jet transverse momentum:

$$
H_{T}=\sum_{\ell, j} p_{T}
$$

The dominant SM backgrounds have been generated in MadGraph and then showered through PYTHIA. Also appropriate $K$-factors were used to match them with the next-to-leading order cross section. We have identified dominant SM backgrounds as: $t \bar{t}, W^{+} W^{-}, W^{ \pm} Z, Z Z, W j$, $Z j$ and Drell-Yan for the chosen signal regions. The discovery potential of the model, in terms of signal significance, is shown for only those cases where the signal can be clearly distinguished from the SM background. In each case, we have also shown the status of the pure IDM scenario for comparison purposes. Once again we would like to remind the readers that the purpose of the collider phenomenology in our context is not to search for signals of the vectorlike fermions, but to look for the signature of the model itself. In that sense, our choice of observables and cuts is different from that of dedicated experimental searches done at the LHC or LEP.

\section{B. Dilepton with missing energy final state}

In Figs. 16 and 17, we have shown respectively the dilepton invariant mass $\left(M_{\ell \ell}\right)$ and $H_{T}$ distributions for the signal, in comparison with the relevant SM background for final states containing opposite sign dilepton and MET. As one can see from Fig. 16, the primary difference in the $M_{\ell \ell}$ distribution between the signal and the background lies in the fact that we observe a long tail in the case of signal, which is not present in the background. The reason being, in case of signal, the source of the leptons in the final state are the heavy new particles apart from the SM gauge bosons. Since these particles are heavier than the SM gauge bosons, hence the leptons in this case are much more boosted than those coming only from the SM gauge bosons. As a result, we see a flatter distribution for $M_{\ell \ell}$ for the signal compared to the SM background (Fig. 16). As an example in Fig. 13, we see that for signal, the leptons in the final state arise not only from SM $Z$ or $W$ but also from the decay of heavy new particles, e.g., $E^{+} \rightarrow H^{0} \ell^{+}$. 


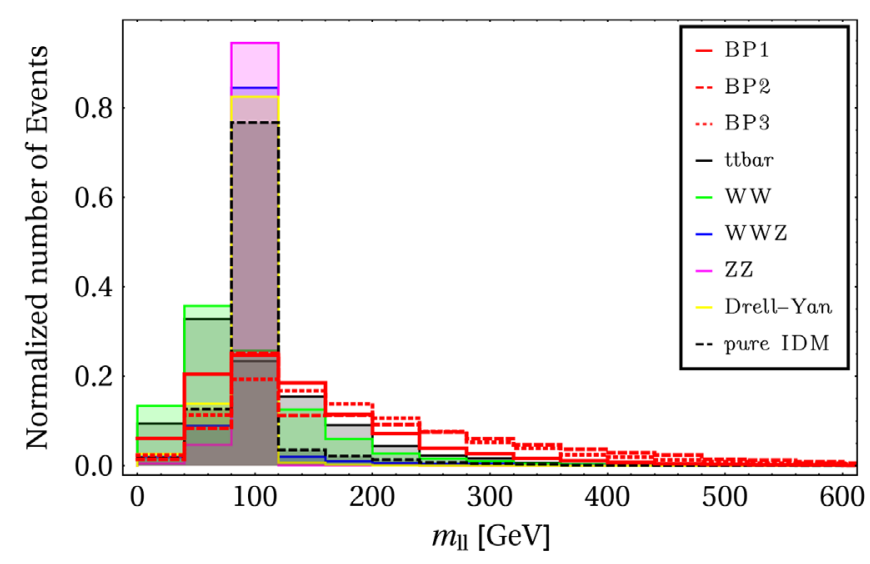

(a)

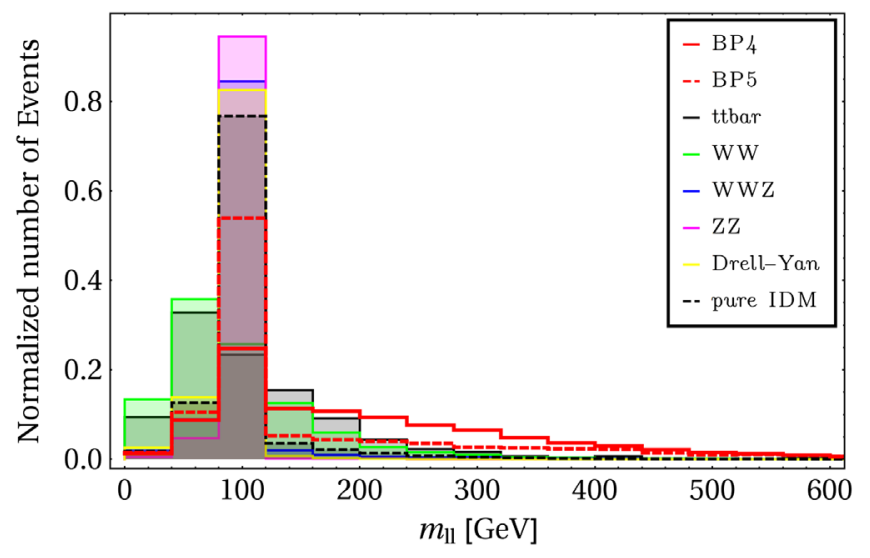

(b)

FIG. 16. Invariant dilepton mass $\left(M_{\ell \ell}\right)$ distribution for the signals (in red), with SM backgrounds for the $\left(\ell^{+} \ell^{-}+\mathbb{E}_{T}\right)$ channel.

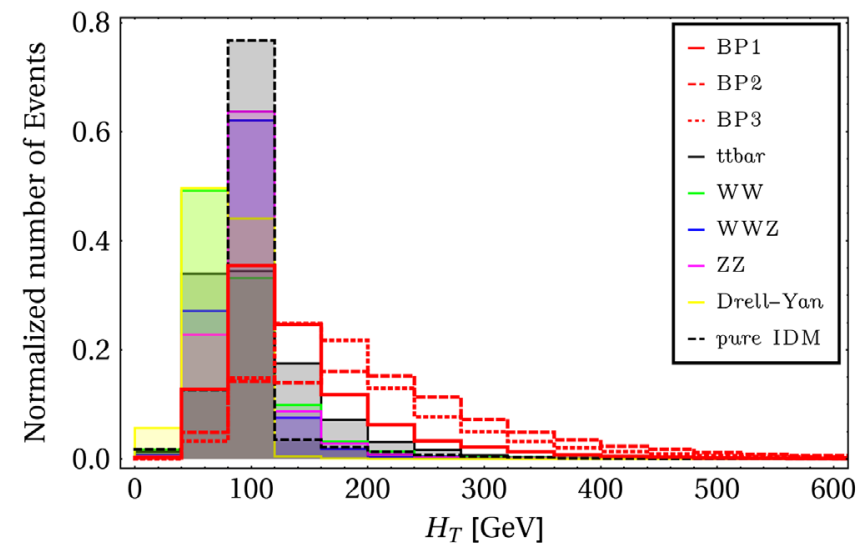

(a)

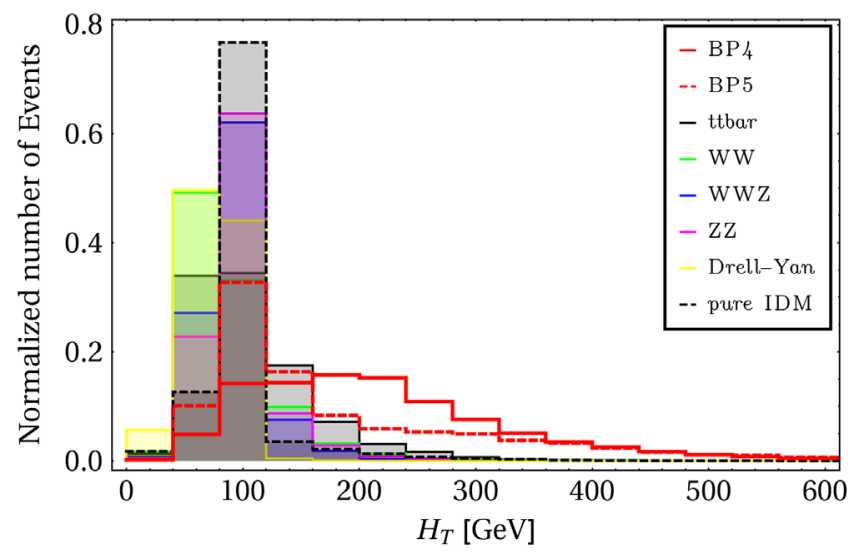

(b)

FIG. 17. $H_{T}$ distribution for the signals (in red), with SM backgrounds $\left(\ell^{+} \ell^{-}+\mathbb{E}_{T}\right)$ channel.

The same argument holds for $H_{T}$, where a flatter signal distribution (Fig. 17) arises because of the less boosted leptons in the final state.

From $M_{\ell \ell}$ distributions shown in Fig. 16 we can see that a cut, $M_{\ell \ell} \gtrsim 300 \mathrm{GeV}$, can help us to get rid of the SM background completely for all BPs except BP1, retaining the signal intact. For BP1, on the other hand, such a cut kills the background along with the signal as well. A similar

TABLE III. Production cross sections for the different signals corresponding to the chosen benchmark points.

\begin{tabular}{lrcc}
\hline \hline$B P$ & $\sigma_{\ell^{+} \ell^{-}+\not L_{T}}(\mathrm{fb})$ & $\sigma_{j j+\not \phi_{T}}(\mathrm{fb})$ & $\sigma_{\ell^{+} \ell^{-}+j j+\not \phi_{T}}(\mathrm{fb})$ \\
\hline 1 & 588 & 278 & 0.31 \\
2 & 54 & 181 & 2.91 \\
3 & 173 & 177 & 2.73 \\
4 & 54 & 172 & 2.75 \\
5 & 17 & 173 & 2.98 \\
6 & 20 & 174 & 2.88 \\
7 & 307 & 174 & 1.00 \\
\hline \hline
\end{tabular}

kind of trend is also observed in the $H_{T}$ distribution. In order to determine the signal significance, we have used $M_{\ell \ell}>200 \mathrm{GeV}$ and $H_{T}>280 \mathrm{GeV}$ cuts for BP1-5.

For all the benchmarks, final state cross section with all of the above-mentioned cuts imposed are tabulated in Table III. Corresponding cross sections for dominant SM

TABLE IV. Final state signal cross section with $M_{\ell \ell}>$ $200 \mathrm{GeV}$ and $H_{T}>280 \mathrm{GeV}$ for the $O S D+\mathscr{E}_{T}$ final state. All simulations are done at $\sqrt{s}=14 \mathrm{TeV}$.

\begin{tabular}{lccc}
\hline \hline $\begin{array}{l}\text { Benchmark } \\
\text { points }\end{array}$ & $\begin{array}{c}\sigma^{\text {production }} \\
(\mathrm{pb})\end{array}$ & $\begin{array}{c}\sigma^{\mathrm{OSD}} \\
(\mathrm{fb})\end{array}$ \\
\hline BP1 & 0.58 & & 11.98 \\
BP2 & 0.05 & $M_{\ell \ell}>200 \mathrm{GeV}$ & 3.05 \\
BP3 & 0.16 & $H_{T}>280 \mathrm{GeV}$ & 8.77 \\
BP4 & 0.05 & & 3.04 \\
BP5 & 0.01 & & 0.45 \\
BP6 & 0.02 & & 0.48 \\
BP7 & 0.30 & & 0.85 \\
Pure IDM & 0.16 & & 0.03 \\
\hline \hline
\end{tabular}


TABLE V. Final state SM background cross section with $M_{\ell \ell}>200 \mathrm{GeV}$ and $H_{T}>280 \mathrm{GeV}$ for the $O S D+E_{T}$ final state. All simulations are done at $\sqrt{s}=14 \mathrm{TeV}$.

\begin{tabular}{lccc}
\hline \hline $\begin{array}{l}\text { Benchmark } \\
\text { points }\end{array}$ & $\begin{array}{c}\sigma^{\text {production }} \\
(\mathrm{pb})\end{array}$ & $\begin{array}{c}\sigma^{\mathrm{OSD}} \\
(\mathrm{fb})\end{array}$ \\
\hline$t \bar{t}$ & 81.64 & & 48.78 \\
$W W$ & 99.98 & $M_{\ell \ell}>200 \mathrm{GeV}$ & 36.99 \\
$W W Z$ & 0.15 & $H_{T}>280 \mathrm{GeV}$ & 0.06 \\
$Z Z$ & 14.01 & & 0.14 \\
Drell-Yan & 2272.80 & & 0.14 \\
\hline \hline
\end{tabular}

backgrounds are also shown in Table IV. In spite of the fact that such a cut kills most of the signals for BP1, due to its high production cross section (as shown in Table V) it shows a very high significance along with BP3. Similarly, $E_{T}>200 \mathrm{GeV}$ and $H_{T}>320 \mathrm{GeV}$ have been used to compute the significance of BP6 and BP7 and a similar excess is seen in the case of BP7 due to its high production cross section. Therefore, the benchmarks BP1, BP3 and $\mathrm{BP} 7$ are prone to be eliminated in ongoing $\mathrm{LHC}$ runs, if no excess is being found in those channels.

The significance plots for $O S D+\mathbb{E}_{T}$ channels are shown in Fig. 18. From the plot we can infer that:

(i) So far at LHC, we have not seen any excess in $\ell^{+} \ell^{-}+\mathbb{E}_{T}$ channels, therefore, our model parameters in BP1 and BP7 are most likely to be ruled out by LHC.

(ii) There is still a possibility that BP3 and BP6 might be probed in the future run of $\mathrm{LHC}$ at a luminosity $\mathcal{L} \sim 60$ and $200 \mathrm{fb}^{-1}$ respectively.

(iii) Due to identical masses of the vectorlike leptons (and hence the same production cross section for dilepton final state), the significance of BP2 and BP4 is exactly the same. Both of them reach a discovery potential of $5 \sigma$ at very high luminosity $\left(\mathcal{L} \sim 700 \mathrm{fb}^{-1}\right)$.

(iv) It will be very hard to distinguish BP5 from pure IDM and it seems almost impossible to be probed within the future limit of LHC luminosity.

Therefore, from the collider searches of the $\left(\ell^{+} \ell^{-}+\right.$ $\mathbb{E}_{T}$ ) final state, it is hard to rule out the model parameters entirely for some specific choices of BPs. What we see is that vectorlike leptons with masses $\lesssim 200 \mathrm{GeV}$ are prone to be ruled out by LHC at the present luminosity. But vectorlike leptons with masses of $\gtrsim 250 \mathrm{GeV}$ and above are yet to be probed by the future high luminosity runs. In the future runs the nonobservance of any excess in the data will help us to rule out higher mass regions of the vectorlike leptons.

\section{Dijet plus $\mathbb{E}_{T}$ final state with and without $b$-tagging}

We have used $\mathbb{E}_{T}$ and $H_{T}$ as observables in order to distinguish the jet final state signal from that of the SM backgrounds. For the SM, the only source of $\mathbb{E}_{T}$ is the SM neutrinos, which can be approximately taken to be massless at the colliders. As a result, the peak of the $\mathbb{E}_{T}$ distribution for the background lies at a very low value of $\mathbb{E}_{T}$. For signal, on the other hand, we can see from Figs. 19 and 20 that except for BP1, in all the other benchmark scenarios it is extremely difficult to separate the signal of our model from the SM background and also from the pure IDM signal. This is due to the fact that in our model the dominant contributions to missing energy are coming from the diagrams in Fig. 14, where final states contain both SM neutrinos and the DM. Hence the shape of the distribution is not just dictated by the massive DM, but the massless neutrinos as well. The final state cross sections are listed in Table VI for the signal where we can see that the cross

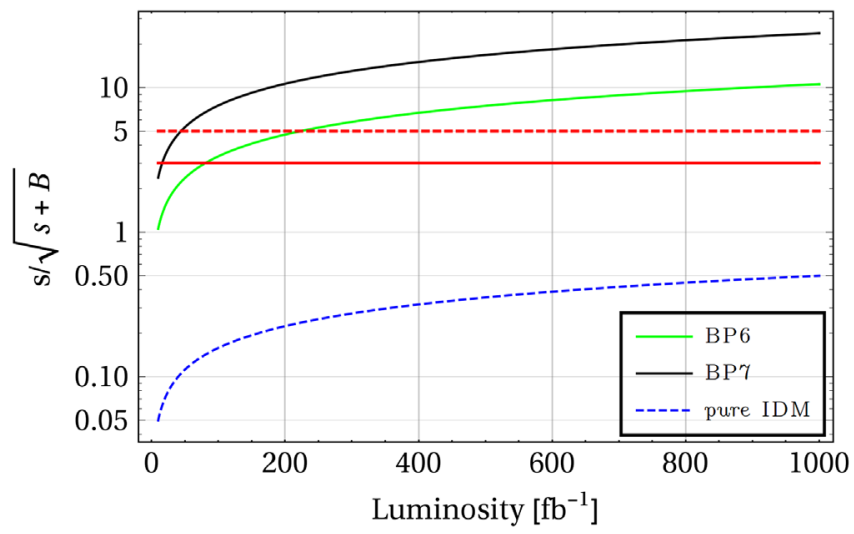

(b)

FIG. 18. Significance plot for dilepton plus $\mathscr{E}_{T}$ final state for the different BPs. The dashed blue line shows the significance for the pure IDM scenario for comparison. The thick red and the dashed red lines are respectively showing the $3 \sigma$ and $5 \sigma$ confidences. Note that we have used $M_{\ell \ell}>200 \mathrm{GeV}$ and $H_{T}>280 \mathrm{GeV}$ cuts to compute the significance of BP1 to BP5 (left plot) while $\mathscr{E}_{T}>200 \mathrm{GeV}$ and $H_{T}>320 \mathrm{GeV}$ have been used to compute the significance of BP6 and BP7 (right plot). 


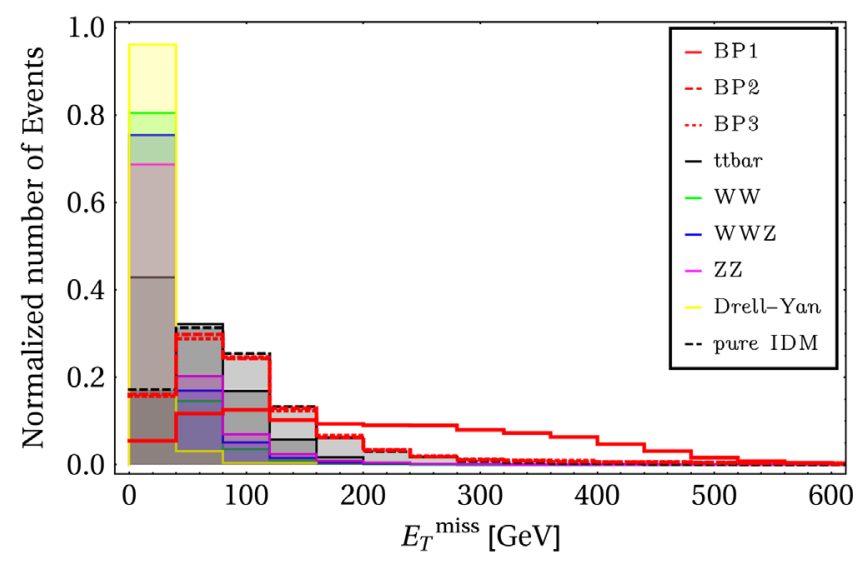

(a)

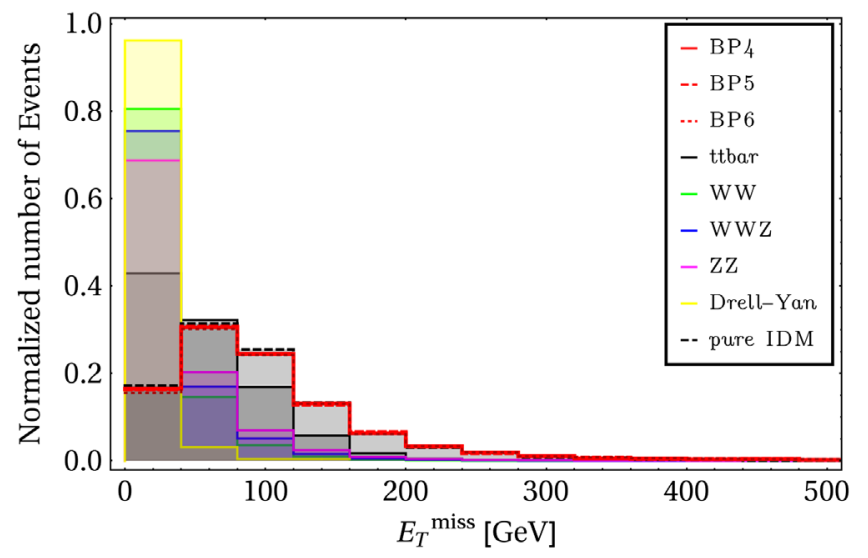

(b)

FIG. 19. Missing energy $\left(\mathscr{E}_{T}\right)$ distribution for the signals (in red), with SM backgrounds for the dijet $+\mathbb{E}_{T}$ channel. We note that in the left plot, it is hard to distinguish the distributions of the benchmark scenarios BP2 and BP3 from each other. Similarly in the right plot all the signal distributions almost overlap with each other.

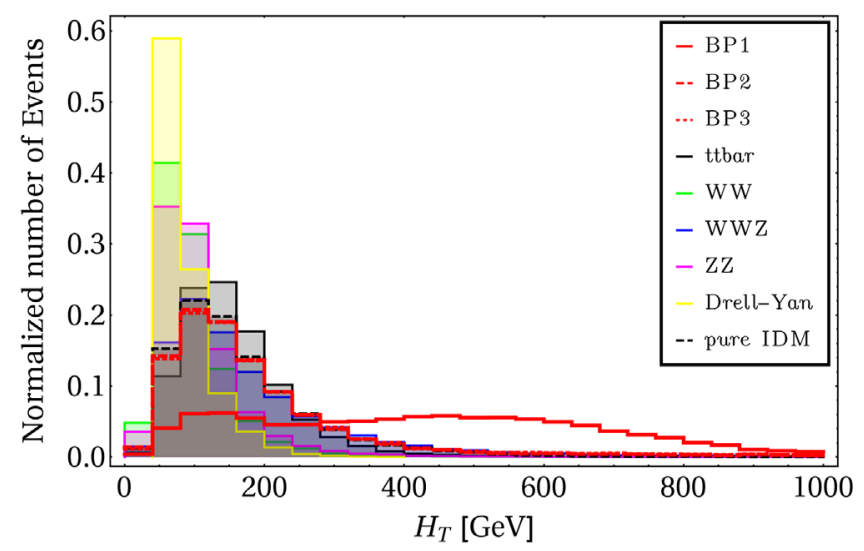

(a)

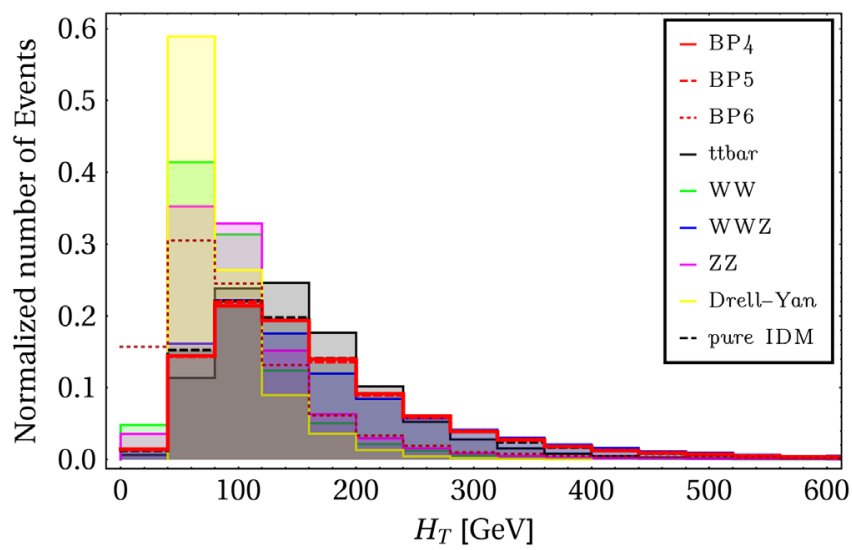

(b)

FIG. 20. $\quad H_{T}$ distribution for the signals (in red), with SM backgrounds for the dijet $+\mathbb{E}_{T}$ channel. We note that in the left plot, it is hard to distinguish the distributions of the benchmark scenarios BP2 and BP3 from each other. Similarly in the right plot all the signal distributions for BP4 and BP5 almost overlap with each other.

TABLE VI. Final state signal cross section with $\mathbb{E}_{T}: 240$ $280 \mathrm{GeV}$ for the dijet $+\mathscr{E}_{T}$ final state. The numbers in the parentheses are the corresponding cross section for exclusive $b$-jet final state. All simulations are done at $\sqrt{s}=14 \mathrm{TeV}$.

\begin{tabular}{|c|c|c|c|}
\hline $\begin{array}{l}\text { Benchmark } \\
\text { points }\end{array}$ & $\begin{array}{c}\sigma^{\text {production }} \\
(\mathrm{pb})\end{array}$ & & $\begin{array}{c}\sigma^{\mathrm{jj}}\left(\sigma^{\mathrm{bb}}\right) \\
(\mathrm{fb})\end{array}$ \\
\hline BP1 & 0.27 & & $7.62(1.87)$ \\
\hline BP2 & 0.18 & & $1.27(0.09)$ \\
\hline BP3 & 0.16 & $\mathbb{E}_{T}: 240-280 \mathrm{GeV}$ & $1.22(0.08)$ \\
\hline BP4 & 0.17 & & $1.29(0.06)$ \\
\hline BP5 & 0.17 & & $1.15(0.05)$ \\
\hline Pure IDM & 0.16 & & $1.14(0.03)$ \\
\hline
\end{tabular}

TABLE VII. Final state signal cross section with $\mathbb{E}_{T}: 240$ $280 \mathrm{GeV}$ for the dijet $+\mathscr{E}_{T}$ final state. The numbers in the parentheses are the corresponding cross section for the exclusive $b$-jet final state. All simulations are done at $\sqrt{s}=14 \mathrm{TeV}$.

\begin{tabular}{|c|c|c|c|}
\hline $\begin{array}{l}\text { Benchmark } \\
\text { points }\end{array}$ & $\begin{array}{c}\sigma^{\text {production }} \\
(\mathrm{pb})\end{array}$ & & $\begin{array}{c}\sigma^{\mathrm{jj}}\left(\sigma^{\mathrm{bb}}\right) \\
(\mathrm{fb})\end{array}$ \\
\hline$t \bar{t}$ & 814.64 & \multirow{4}{*}{$\mathscr{E}_{T}: 240-280 \mathrm{GeV}$} & $317.71(24.43)$ \\
\hline$W W$ & 99.98 & & $12.99(0.00)$ \\
\hline$W W Z$ & 0.15 & & $0.14(0.00)$ \\
\hline$Z Z$ & 14.01 & & $11.76(0.28)$ \\
\hline
\end{tabular}




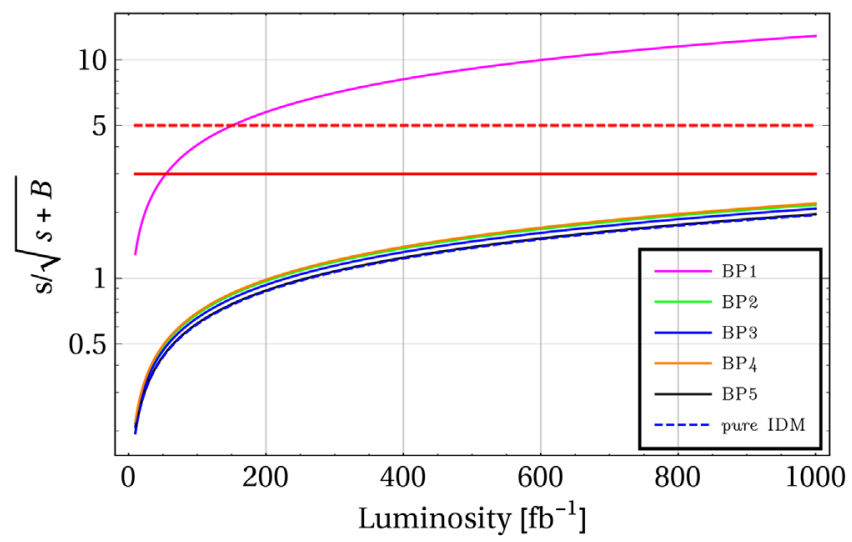

(a)

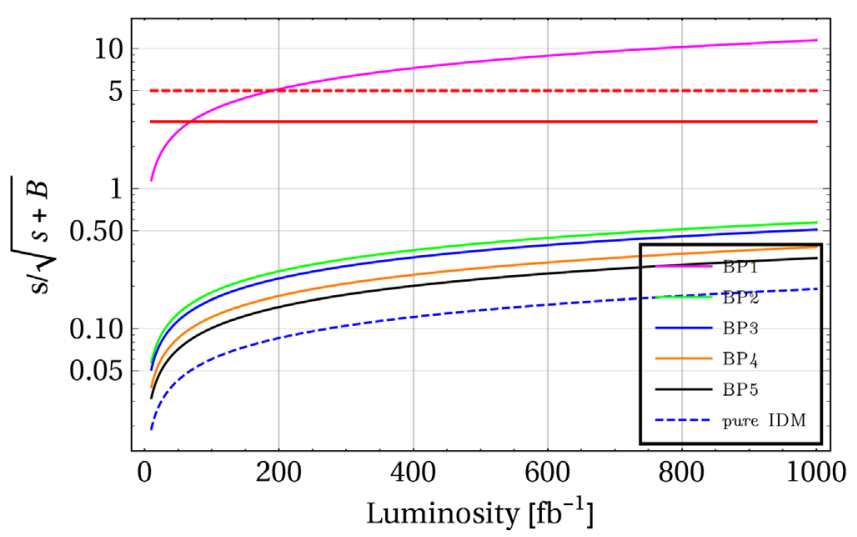

(b)

FIG. 21. Left: Significance plot for the dijet $+\mathscr{E}_{T}$ final state for different BPs. Right: The same when only $b$-jets are considered. In both plots, the dashed blue line shows the significance for the pure IDM scenario; the thick red and the dashed red lines are respectively showing the $3 \sigma$ and $5 \sigma$ confidences. We have refrained from showing the significance plots for BP6 and BP7 because they were similar and very close to BP3-5, making the plot look messy.

section for BP1 is significantly large, while for other benchmarks they are almost the same. For the SM backgrounds corresponding cross sections are also tabulated in Table VII. Note that, due to hadronic final state, the backgrounds are more vigorous here, making the signal less significant than that of the leptonic final state.

In Fig. 21, we have shown the significance of different BPs for the dijet final state with (left) and without (right) $b$ tagging. For BP1, a significant excess can be seen in all the distributions and it shows a very high significance for the present LHC luminosity, which is very likely to be ruled out by LHC data. So far, no excess has been found in the present LHC run [65]. For inclusive dijet search, all other BPs reach a $5 \sigma$ significance for a luminosity $\mathcal{L} \sim 600 \mathrm{fb}^{-1}$, as shown in the lhs of Fig. 21. It is also impossible to distinguish BP5 from the pure IDM scenario, while all other BPs (except BP1) lie very close to each other because of comparable production cross sections. On the other hand, for exclusive $b$-tagged final states, although the BPs can be distinguished from the pure IDM case, none of them reach a discovery limit even at very high luminosity. This makes the model impossible to be probed at the LHC for final states containing $b$-jets. Thus, we can infer that, at the present LHC luminosity the nonobservation of any excess rules out the vectorlike quark with masses $\leq 500 \mathrm{GeV}$ (BP1). However, $M_{D}$ in between 700 and $900 \mathrm{GeV}$ are still allowed which can further be constrained if we do not see any excess in the dijet plus $\mathbb{E}_{T}$ signal at high luminosity LHC runs. We have also noted that $M_{D} \gtrsim 900 \mathrm{GeV}$ cannot be ruled out at the LHC even at a very high luminosity run. This makes the model difficult to be probed in the dijet plus $\mathbb{E}_{T}$ final state even at high luminosity LHC runs.

In passing we would like to mention that dilepton plus dijet with missing energy final state has production cross section lower than that of other final state signatures we discussed so far (as tabulated in Table III) which will result in very low signal significance over background. Therefore, we refrain from elaborating the fate of such final states at the LHC.

\section{RENORMALIZATION GROUP EQUATION (RGE) RUNNING OF THE COUPLINGS}

Any finite coupling is expected to hit a Landau pole at some scale. The Landau pole is the scale where the couplings become infinite. We check the high scale validity of our model by solving the RGEs using PyR@TE2 [66]. The RG equations for pure IDM have been studied extensively before [67], but the addition of vectorlike particles will modify the gauge, quartic and Yukawa couplings as given below. We have considered the running of the SM Yukawas and gauge couplings at the two-loop level. The running of all the new couplings are considered at one-loop level.

The RG equations can be in general written as

$$
\frac{d \lambda}{d \mu} \equiv \beta_{\lambda}=\beta_{\lambda}^{I}+\beta_{\lambda}^{I I}
$$

where $\lambda$ is any arbitrary coupling, $\mu$ is the energy scale and $\beta^{I(I I)}$ is the one(two)-loop beta function. In this section we present the one-loop RG evolution equations for the gauge, quartic and Yukawa couplings.

The evolution of the gauge couplings is given by

$$
\begin{gathered}
(4 \pi)^{2} \beta_{g_{s}}^{I}=-6 g_{s}^{3}, \quad(4 \pi)^{2} \beta_{g}^{I}=-3 g^{3}, \\
(4 \pi)^{2} \beta_{g_{1}}^{I}=+\frac{35}{3} g_{1}^{3},
\end{gathered}
$$

where, $g_{s}, g$ and $g_{1}$ are the $\mathrm{SU}(3), \mathrm{SU}(2)$ and $\mathrm{U}(1)$ gauge couplings respectively. 
The RG equations for the quartic couplings are given by

$$
\begin{aligned}
(4 \pi)^{2} \beta_{\lambda_{1}}^{I}= & -24 Y_{t}^{2} Y_{b}^{2}-12 Y_{b}^{4}-12 Y_{t}^{4}+4 \lambda_{3}^{2}+2 \lambda_{4}^{2}+2 \lambda_{5}^{2}+12 \lambda_{1}^{2}+4 \lambda_{3} \lambda_{4}-3 \lambda_{1} g_{1}^{2} \\
& -9 \lambda_{1} g^{2}+\frac{3 g_{1}^{4}}{4}+\frac{9 g^{4}}{4}+\frac{3}{2} g^{2} g_{1}^{2}-4 Y_{\tau}^{4}+4 \lambda_{1} Y_{\tau}^{2} \\
(4 \pi)^{2} \beta_{\lambda_{2}}^{I}= & -12 \lambda_{b}^{2}+2 \lambda_{4}^{2}+2 \lambda_{5}^{2}-4 \lambda_{\mu}^{4}-4 \lambda_{\tau}^{4}-8 \lambda_{\mu}^{2} \lambda_{\tau}^{2}+12 \lambda_{2}^{2}+4 \lambda_{3}^{2}+4 \lambda_{3} \lambda_{4}+4 \lambda_{2} \lambda_{\mu}^{2} \\
& +4 \lambda_{2} \lambda_{\tau}^{2}+\frac{3 g_{1}^{4}}{4}+\frac{9 g^{4}}{4}+\frac{3}{2} g^{2} g_{1}^{2}-3 \lambda_{2} g_{1}^{2}-9 \lambda_{2} g^{2}+12 \lambda_{2} \lambda_{b}^{2} \\
(4 \pi)^{2} \beta_{\lambda_{3}}^{I}= & +4 \lambda_{3}^{2}+2 \lambda_{4}^{2}+2 \lambda_{5}^{2}+2 \lambda_{1} \lambda_{4}+2 \lambda_{2} \lambda_{4}+6 \lambda_{1} \lambda_{3}+6 \lambda_{2} \lambda_{3}+\frac{3 g_{1}^{4}}{4}+\frac{9 g^{4}}{4}-\frac{3}{2} g^{2} g_{1}^{2} \\
& -9 \lambda_{3} g^{2}-3 \lambda_{3} g_{1}^{2}+2 \lambda_{3} Y_{\tau}^{2}+2 \lambda_{3} \lambda_{\mu}^{2}+2 \lambda_{3} \lambda_{\tau}^{2}+6 \lambda_{3} Y_{b}^{2}+6 \lambda_{3} Y_{t}^{2}+6 \lambda_{3} \lambda_{b}^{2} \\
(4 \pi)^{2} \beta_{\lambda_{5}}^{I}= & +2 \lambda_{1} \lambda_{5}+2 \lambda_{2} \lambda_{5}+8 \lambda_{3} \lambda_{5}+12 \lambda_{3} \lambda_{5}-9 \lambda_{5} g^{2}-3 \lambda_{5} g_{1}^{2}+2 \lambda_{5} Y_{\tau}^{2}+2 \lambda_{5} \lambda_{\mu}^{2} \\
& +2 \lambda_{5} \lambda_{\tau}^{2}+6 \lambda_{5} Y_{b}^{2}+6 \lambda_{5} Y_{t}^{2}+6 \lambda_{5} \lambda_{b}^{2} \\
(4 \pi)^{2} \beta_{\lambda_{45}}^{I}= & -12 \lambda_{b}^{2} Y_{b}^{2}-12 \lambda_{b}^{2} Y_{t}^{2}+4 \lambda_{4}^{2}+8 \lambda_{5}^{2}-4 \lambda_{\mu}^{2} Y_{\tau}^{2}-4 \lambda_{\tau}^{2} Y_{\tau}^{2}+2 \lambda_{1} \lambda_{4}+2 \lambda_{1} \lambda_{5}-9 \lambda_{4} g^{2}-3 \lambda_{4} g_{1}^{2}-9 \lambda_{5} g^{2}-3 \lambda_{5} g_{1}^{2} \\
& +2 \lambda_{2} \lambda_{4}+2 \lambda_{2} \lambda_{5}+2 \lambda_{4} Y_{\tau}^{2}+2 \lambda_{4} \lambda_{\mu}^{2}+2 \lambda_{4} \lambda_{\tau}^{2}+2 \lambda_{5} Y_{\tau}^{2}+2 \lambda_{5} \lambda_{\mu}^{2}+2 \lambda_{5} \lambda_{\tau}^{2}+3 g_{1}^{2} g^{2}+6 \lambda_{4} Y_{t}^{2}+6 \lambda_{4} Y_{b}^{2} \\
& +6 \lambda_{4} \lambda_{b}^{2}+6 \lambda_{5} Y_{t}^{2}+6 \lambda_{5} Y_{b}^{2}+6 \lambda_{5} \lambda_{b}^{2}+8 \lambda_{3} \lambda_{4}+8 \lambda_{3} \lambda_{5}+12 \lambda_{4} \lambda_{5}
\end{aligned}
$$

where $\lambda_{45}=\lambda_{4}+\lambda_{5}$.

The RG equations for the Yukawa couplings are given by

$$
\begin{aligned}
(4 \pi)^{2} \beta_{Y_{t}}^{I}= & Y_{t}\left(\frac{1}{2} \lambda_{b}^{2}+\frac{9}{2} Y_{b}^{2}+\frac{9}{2} Y_{t}^{2}+Y_{\tau}^{2}-8 g_{s}^{2}-\frac{9}{4} g^{2}-\frac{5}{12} g_{1}^{2}\right) \\
(4 \pi)^{2} \beta_{Y_{b}}^{I}= & Y_{b}\left(\frac{1}{2} \lambda_{b}^{2}+\frac{9}{2} Y_{b}^{2}+\frac{9}{2} Y_{t}^{2}+Y_{\tau}^{2}-8 g_{s}^{2}-\frac{9}{4} g^{2}-\frac{17}{12} g_{1}^{2}\right) \\
(4 \pi)^{2} \beta_{Y_{\tau}}^{I}= & Y_{\tau}\left(\frac{1}{2} \lambda_{\mu}^{2}+\frac{1}{2} \lambda_{\tau}^{2}+3 Y_{b}^{2}+3 Y_{t}^{2}\right. \\
& \left.+\frac{5}{2} Y_{\tau}^{2}-\frac{9}{4} g^{2}-\frac{15}{4} g_{1}^{2}\right) \\
(4 \pi)^{2} \beta_{\lambda_{b}}^{I}= & \lambda_{b}\left(\frac{9}{2} \lambda_{b}^{2}+\frac{1}{2} Y_{b}^{2}+\frac{1}{2} Y_{t}^{2}+\lambda_{\mu}^{2}+\lambda_{\tau}^{2}\right. \\
& \left.-8 g_{s}^{2}-\frac{9}{4} g^{2}-\frac{5}{12} g_{1}^{2}\right) \\
(4 \pi)^{2} \beta_{\lambda_{\mu}}^{I}= & \lambda_{\mu}\left(\frac{5}{2} \lambda_{\mu}^{2}+\frac{5}{2} \lambda_{\tau}^{2}+3 \lambda_{b}^{2}+\frac{1}{2} Y_{\tau}^{2}-\frac{9}{4} g^{2}-\frac{15}{4} g_{1}^{2}\right) \\
(4 \pi)^{2} \beta_{\lambda_{\tau}}^{I}= & \lambda_{\tau}\left(\frac{5}{2} \lambda_{\mu}^{2}+\frac{5}{2} \lambda_{\tau}^{2}+3 \lambda_{b}^{2}+\frac{1}{2} Y_{\tau}^{2}-\frac{9}{4} g^{2}-\frac{15}{4} g_{1}^{2}\right) .
\end{aligned}
$$

In Fig. 22, we have shown the variation of the Yukawa couplings in our model for the allowed benchmark scenarios. We note that the Landau pole is reached at $\sim 10^{7} \mathrm{GeV}$ for both BP2 and BP4, for BP5 the Landau pole is reached at $\gtrsim 10^{6} \mathrm{GeV}$, while for BP6, it is reached at $10^{5} \mathrm{GeV}$. We note that the cutoff scale is dependent on the initial value of our NP couplings. The cutoff scale is higher if any one of the couplings is as small as in BP2 (here the smaller one being $\lambda_{\mu}=0.7$ while the largest one being $\lambda_{\tau}=1.2$ ) while for BP6 in which both $\lambda_{\mu}$ and $\lambda_{\tau}$ equal 1.5 and $\lambda_{b}=0.1$, the Landau pole is reached at $10^{5} \mathrm{GeV}$. Thus the Landau pole is mainly driven by the smaller coupling and since we have chosen $\lambda_{\tau}$ to be high in all the cases, the perturbativity of

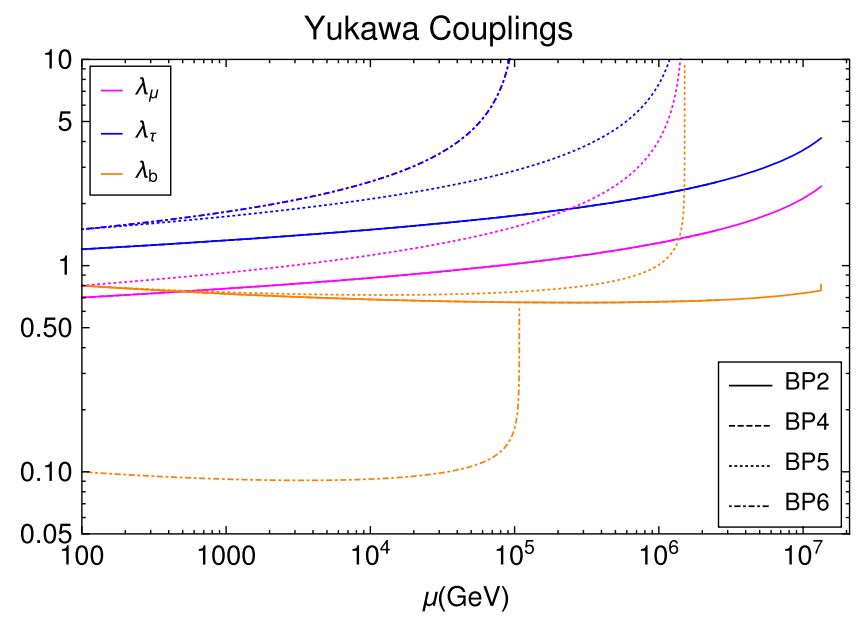

FIG. 22. Running of the NP Yukawa couplings of our model with energy scale $\mu$ (in $\mathrm{GeV}$ ) is shown here. The magenta lines represent the evolution of $\lambda_{\mu}$, the blue ones represent that of $\lambda_{\tau}$ while the orange ones are for $\lambda_{b}$. The solid, dashed, dotted and dot-dashed styles for the legends are used to represent the running for benchmark points 2, 4, 5 and 6 respectively. Note that the solid and dashed lines overlap (due to similar variation of the couplings) and hence are indistinguishable. Also the magenta and blue dot-dashed lines for BP6 overlap due to similar coupling strengths. 
our theory is mostly determined by the value either $\lambda_{\mu}$ or $\lambda_{b}$, whichever is smaller.

\section{SUMMARY}

In this paper we have studied an extension of the inert Higgs doublet model with vectorlike fermion singlets under $S U(2)_{L}$ gauge symmetry. The model offers a DM candidate the same as that in pure IDM, and the vectorlike fermions act as mediators between the dark sector and the visible sector, apart from the usual SM Higgs portal interactions. Due to the presence of the Yukawa interaction and familydependent couplings, we can now have interesting FCNC and LFV processes, which can explain anomalies in $R\left(K^{(*)}\right)$ data and also have the potential to incorporate the observed data on muon $(g-2)$. At the moment, satisfying all the other constraints, our model can comfortably explain the lower limit of the observed discrepancy in muon $(g-2)$. We have to wait for a more precise estimate of $a_{\mu}$ for the final verdict. We have studied the parameter space of the model in detail considering bounds from DM relic density, direct detection, as well as from flavor data for both the low and the high mass region of the DM. Apart from change in allowed DM mass values from relic density requirements, due to the existence of new interactions mediated by vectorlike fermions, we also find more promising direct detection rates compared to pure IDM, for chosen benchmark points.

From the resulting constrained parameter space, we have chosen a set of benchmark points for further collider studies. Because of the presence of the exotic particles, this model gives rise to several interesting signals in collider consisting of hadronically quiet dilepton channel $\left(\ell^{+} \ell^{-}+\mathscr{E}_{T}\right)$, dijet channel $\left(j j+\mathscr{E}_{T}\right)$ and dilepton plus dijet channel $\left(\ell^{+} \ell^{-}+j j+\mathscr{E}_{T}\right)$, along with missing energy. Final states containing two leptons with two jets plus missing energy provide a very small production cross section, and we refrain from analyzing such signals in our work. Of the other two channels, the hadronically quiet dilepton final state shows a $5 \sigma$ significance in a few of our chosen benchmark scenarios (BP2 and BP4) with $M_{E_{2}}$ and
$M_{E_{3}} \gtrsim 200 \mathrm{GeV}$ for $M_{\ell \ell}>200 \mathrm{GeV}$ and $H_{T}>280 \mathrm{GeV}$, and the required luminosity is $\sim 700 \mathrm{fb}^{-1}$. BP6 is also likely to be probed at the future run of the LHC when the luminosity reaches $\sim 200 \mathrm{fb}^{-1}$. We note that the vectorlike lepton masses $\lesssim 200 \mathrm{GeV}$ are very likely ruled out by LHC data since they have not seen any excess in the abovementioned final states at the present luminosity. For the inclusive dijet plus $\mathscr{E}_{T}$ final state, on the other hand, a $5 \sigma$ discovery can be claimed in a couple of benchmark scenarios with $M_{D} \gtrsim 600 \mathrm{GeV}$ for luminosity $\geq 600 \mathrm{fb}^{-1}$.

To summarize, the vectorlike fermion extension of IDM is capable of explaining anomalous results like $R\left(K^{(*)}\right)$ and muon $(g-2)$; the required new parameter spaces are allowed by other flavor data like the rare and radiative $B_{q}(q=d$, s $)$ decays, $B_{q}-\bar{B}_{q}$ mixing and the LFV decays like $\tau \rightarrow \mu \gamma, \mu \rightarrow e \gamma$ etc. The DM of the model satisfies Planck-observed relic density, obeying bounds from recent direct search data. The model can also be probed in the LHC experiment for a higher luminosity for some particular final states satisfying all the constraints mentioned above. We also check the perturbative unitarity of the model and find that for the chosen benchmark points the model can remain perturbative up to an energy scale $10^{5}-10^{7} \mathrm{GeV}$.

\section{ACKNOWLEDGMENTS}

B. B. and L. M. would like to thank Subhadeep Mondal and Subhaditya Bhattacharya for useful discussions on collider analysis. D. B. acknowledges the support from IIT Guwahati start-up grant (Reference No. xPHYSUGIITG01152xxDB001). We would like to thank Tanmoy Mondal for his useful comments. S. N. acknowledges the support from a research grant by the Science and Engineering Research Board, Government of India, under Grant No. CRG/2018/001260.

\section{APPENDIX: TWO LOOP RG EQUATIONS}

The two-loop RG equation for the SM gauge couplings is given by

$$
\begin{aligned}
& \beta_{g_{s}}^{I I}=\frac{g_{s}^{3}}{(4 \pi)^{4}}\left(-7 g_{s}^{2}-2 Y_{b}^{2}-2 Y_{t}^{2}+\frac{13}{6} g_{1}^{2}+\frac{9}{2} g^{2}-2 \lambda_{b}^{2}\right) \\
& \beta_{g}^{I I}=\frac{g^{3}}{(4 \pi)^{4}}\left(8 g^{2}+2 g_{1}^{2}+12 g_{s}^{2}-\frac{3}{2} Y_{b}^{2}-\frac{3}{2} Y_{t}^{2}-\frac{3}{2} \lambda_{b}^{2}-\frac{1}{2} Y_{\tau}^{2}-\frac{1}{2} \lambda_{\mu}^{2}-\frac{1}{2} \lambda_{\tau}^{2}\right) \\
& \beta_{g_{1}}^{I I}=\frac{g_{1}^{3}}{(4 \pi)^{4}}\left(\frac{214}{9} g_{1}^{2}+6 g^{2}+\frac{52}{3} g_{s}^{2}-\frac{17}{6} Y_{b}^{2}-\frac{5}{6} Y_{t}^{2}-\frac{5}{2} Y_{\tau}^{2}-\frac{5}{2} \lambda_{\mu}^{2}-\frac{5}{2} \lambda_{\tau}^{2}-\frac{5}{6} \lambda_{b}^{2}\right) .
\end{aligned}
$$


The two-loop RG equations for the SM Yukawa couplings are given by

$$
\begin{aligned}
& \beta_{Y_{t}}^{I I}=\frac{Y_{t}}{(4 \pi)^{4}}\left(-12 Y_{t}^{4}-12 Y_{b}^{4}-24 Y_{b}^{2} Y_{t}^{2}-\frac{5}{2} Y_{b}^{2} \lambda_{b}^{2}-\frac{5}{2} Y_{t}^{2} \lambda_{b}^{2}-\frac{5}{2} \lambda_{b}^{4}+\lambda_{3}^{2}+\lambda_{4}^{2}+\lambda_{3} \lambda_{4}+\frac{3}{2} \lambda_{1}^{2}+\frac{3}{2} \lambda_{5}^{2}-6 \lambda_{1} Y_{b}^{2}\right. \\
& -6 \lambda_{1} Y_{t}^{2}-4 \lambda_{4} \lambda_{b}^{2}-2 \lambda_{3} \lambda_{b}^{2}+16 g_{s}^{2} Y_{b}^{2}+16 g_{s}^{2} Y_{t}^{2}-\frac{304}{3} g_{s}^{4}-\frac{47}{72} g_{1}^{4}-\frac{21}{4} g^{4}-\frac{9}{4} Y_{\tau}^{2} Y_{b}^{2}-\frac{9}{4} Y_{\tau}^{2} Y_{t}^{2} \\
& -\frac{9}{4} Y_{\tau}^{4}-\frac{3}{4} \lambda_{\mu}^{2} \lambda_{b}^{2}-\frac{3}{4} \lambda_{\tau}^{2} \lambda_{b}^{2}-\frac{3}{4} Y_{\tau}^{2} \lambda_{\mu}^{2}-\frac{3}{4} Y_{\tau}^{2} \lambda_{\tau}^{2}+\frac{16}{3} g_{s}^{2} \lambda_{b}^{2}+\frac{29}{16} g_{1}^{2} Y_{b}^{2}+\frac{33}{16} g^{2} \lambda_{b}^{2}+\frac{135}{16} g^{2} Y_{b}^{2} \\
& +\frac{135}{16} g^{2} Y_{t}^{2}+\frac{187}{48} g_{1}^{2} Y_{t}^{2}+\frac{247}{144} g_{1}^{2} \lambda_{b}^{2}+9 g^{2} g_{s}^{2}+20 g_{s}^{2} Y_{b}^{2}+20 g_{s}^{2} Y_{t}^{2}-\frac{9}{4} g_{1}^{2} g^{2}+\frac{15}{8} g^{2} Y_{\tau}^{2}+\frac{25}{8} g_{1}^{2} Y_{\tau}^{2} \\
& \left.+\frac{25}{24} g_{1}^{2} Y_{t}^{2}+\frac{31}{9} g_{1}^{2} g_{s}^{2}+\frac{45}{8} g^{2} Y_{b}^{2}+\frac{45}{8} g^{2} Y_{t}^{2}+\frac{85}{24} g_{1}^{2} Y_{b}^{2}\right) \\
& \beta_{Y_{b}}^{I I}=\frac{Y_{b}}{(4 \pi)^{4}}\left(-12 Y_{b}^{4}-12 Y_{t}^{4}-24 Y_{b}^{2} Y_{t}^{2}-\frac{5}{2} \lambda_{b}^{2} Y_{b}^{2}-\frac{5}{2} \lambda_{b}^{2} Y_{t}^{2}-\frac{5}{2} \lambda_{b}^{4}+\lambda_{3}^{2}+\lambda_{4}^{2}+\lambda_{3} \lambda_{4}+\frac{3}{2} \lambda_{1}^{2}+\frac{3}{2} \lambda_{5}^{2}-6 \lambda_{1} Y_{b}^{2}\right. \\
& -6 \lambda_{1} Y_{t}^{2}-4 \lambda_{4} \lambda_{b}^{2}-2 \lambda_{3} \lambda_{b}^{2}+16 g_{s}^{2} Y_{b}^{2}+16 g_{s}^{2} Y_{t}^{2}-\frac{304}{3} g_{s}^{4}-\frac{41}{144} g_{1}^{2} \lambda_{b}^{2}-\frac{21}{4} g^{4}-\frac{9}{4} Y_{\tau}^{2} Y_{b}^{2}-\frac{9}{4} Y_{\tau}^{2} Y_{t}^{2} \\
& -\frac{9}{4} Y_{\tau}^{4}-\frac{3}{4} \lambda_{\mu}^{2} \lambda_{b}^{2}-\frac{3}{4} \lambda_{\tau}^{2} \lambda_{b}^{2}-\frac{3}{4} \lambda_{\mu}^{2} Y_{\tau}^{2}-\frac{3}{4} \lambda_{\tau}^{2} Y_{\tau}^{2}+\frac{16}{3} g_{s}^{2} \lambda_{b}^{2}+\frac{33}{16} g^{2} \lambda_{b}^{2}+\frac{35}{48} g_{1}^{2} Y_{t}^{2}+\frac{135}{16} g^{2} Y_{b}^{2} \\
& +\frac{135}{16} g^{2} Y_{t}^{2}+\frac{223}{48} g_{1}^{2} Y_{b}^{2}+9 g^{2} g_{s}^{2}+20 g_{s}^{2} Y_{b}^{2}+20 g_{s}^{2} Y_{t}^{2}+\frac{77}{8} g_{1}^{4}-\frac{3}{4} g_{1}^{2} g^{2}+\frac{15}{8} g^{2} Y_{\tau}^{2}+\frac{19}{9} g_{1}^{2} g_{s}^{2} \\
& \left.+\frac{25}{8} g_{1}^{2} Y_{\tau}^{2}+\frac{25}{24} g_{1}^{2} Y_{t}^{2}+\frac{45}{8} g^{2} Y_{b}^{2}+\frac{45}{8} g^{2} Y_{t}^{2}+\frac{85}{24} g_{1}^{2} Y_{b}^{2}\right) \\
& \beta_{Y_{\tau}}^{I I}=\frac{Y_{\tau}}{(4 \pi)^{4}}\left(-\frac{3}{4} Y_{\tau}^{4}-\lambda_{\mu}^{2} Y_{\tau}^{2}-\lambda_{\tau}^{2} Y_{\tau}^{2}-2 \lambda_{\mu}^{2} \lambda_{\tau}^{2}-\lambda_{\mu}^{4}-\lambda_{\tau}^{4}+\lambda_{3}^{2}+\lambda_{4}^{2}+\lambda_{3} \lambda_{4}+\frac{3}{2} \lambda_{1}^{2}+\frac{3}{2} \lambda_{5}^{2}-6 \lambda_{1} Y_{\tau}^{2}-4 \lambda_{4} \lambda_{\mu}^{2}\right. \\
& -4 \lambda_{4} \lambda_{\tau}^{2}-2 \lambda_{3} \lambda_{\mu}^{2}-2 \lambda_{3} \lambda_{\tau}^{2}-\frac{27}{2} Y_{t}^{2} Y_{b}^{2}-\frac{27}{4} Y_{b}^{2} Y_{\tau}^{2}-\frac{27}{4} Y_{t}^{2} Y_{\tau}^{2}-\frac{27}{4} Y_{t}^{4}-\frac{27}{4} Y_{b}^{4} \frac{21}{4} g^{4}-\frac{9}{4} \lambda_{b}^{2} Y_{b}^{2} \\
& -\frac{9}{4} \lambda_{b}^{2} Y_{t}^{2}+\frac{33}{16} g^{2} \lambda_{\mu}^{2}+\frac{33}{16} g^{2} \lambda_{\tau}^{2}+\frac{103}{16} g_{1}^{2} \lambda_{\mu}^{2}+\frac{103}{16} g_{1}^{2} \lambda_{\tau}^{2}+\frac{129}{16} g_{1}^{2} Y_{\tau}^{2}+\frac{135}{16} g^{2} Y_{\tau}^{2}+\frac{791}{24} g_{1}^{4} \\
& \left.+20 g_{s}^{2} Y_{b}^{2}+20 g_{s}^{2} Y_{t}^{2}+\frac{9}{4} g_{1}^{2} g^{2}+\frac{15}{8} g^{2} Y_{\tau}^{2}+\frac{25}{8} g_{1}^{2} Y_{\tau}^{2}+\frac{25}{24} g_{1}^{2} Y_{t}^{2}+\frac{45}{8} g^{2} Y_{b}^{2}+\frac{45}{8} g^{2} Y_{t}^{2}+\frac{85}{24} g_{1}^{2} Y_{b}^{2}\right) \text {. }
\end{aligned}
$$

[1] R. Aaij et al. (LHCb Collaboration), Phys. Rev. Lett. 122, 191801 (2019).

[2] R. Aaij et al. (LHCb Collaboration), J. High Energy Phys. 08 (2017) 055.

[3] G. Hiller and F. Kruger, Phys. Rev. D 69, 074020 (2004).

[4] M. Bordone, G. Isidori, and A. Pattori, Eur. Phys. J. C 76, 440 (2016).

[5] A. Abdesselam et al. (Belle Collaboration), arXiv:1904 .02440 .

[6] A. Abdesselam et al., arXiv:1908.01848.

[7] M. Tanabashi et al. (Particle Data Group), Phys. Rev. D 98, 030001 (2018).

[8] P. A. R. Ade et al. (Planck Collaboration), Astron. Astrophys. 594, A13 (2016).
[9] F. Zwicky, Helv. Phys. Acta 6, 110 (1933); Gen. Relativ. Gravit. 41, 207 (2009).

[10] V. C. Rubin and W. K. Ford, Jr., Astrophys. J. 159, 379 (1970).

[11] D. Clowe, M. Bradac, A. H. Gonzalez, M. Markevitch, S. W. Randall, C. Jones, and D. Zaritsky, Astrophys. J. 648, L109 (2006).

[12] M. Taoso, G. Bertone, and A. Masiero, J. Cosmol. Astropart. Phys. 03 (2008) 022.

[13] G. Arcadi, M. Dutra, P. Ghosh, M. Lindner, Y. Mambrini, M. Pierre, S. Profumo, and F. S. Queiroz, Eur. Phys. J. C 78, 203 (2018).

[14] J. Kawamura, S. Okawa, and Y. Omura, Phys. Rev. D 96, 075041 (2017). 
[15] S. Baek, Phys. Lett. B 781, 376 (2018).

[16] D. A. Sierra, F. Staub, and A. Vicente, Phys. Rev. D 92, 015001 (2015).

[17] J. M. Cline, Phys. Rev. D 97, 015013 (2018).

[18] J. M. Cline and J. M. Cornell, Phys. Lett. B 782, 232 (2018).

[19] L. S. Geng, B. Grinstein, S. Jäger, J. M. Camalich, X. L. Ren, and R. X. Shi, Phys. Rev. D 96, 093006 (2017).

[20] N. G. Deshpande and E. Ma, Phys. Rev. D 18, 2574 (1978).

[21] E. Ma, Phys. Rev. D 73, 077301 (2006).

[22] M. Cirelli, N. Fornengo, and A. Strumia, Nucl. Phys. B753, 178 (2006).

[23] L. Lopez Honorez, E. Nezri, J. F. Oliver, and M. H. G. Tytgat, J. Cosmol. Astropart. Phys. 02 (2007) 028.

[24] L. L. Honorez and C. E. Yaguna, J. High Energy Phys. 09 (2010) 046.

[25] L. L. Honorez and C. E. Yaguna, J. Cosmol. Astropart. Phys. 01 (2011) 002.

[26] A. Arhrib, Y. L. S. Tsai, Q. Yuan, and T. C. Yuan, J. Cosmol. Astropart. Phys. 06 (2014) 030.

[27] A. Dasgupta and D. Borah, Nucl. Phys. B889, 637 (2014).

[28] D. Borah, S. Sadhukhan, and S. Sahoo, Phys. Lett. B 771, 624 (2017).

[29] D. Borah and A. Gupta, Phys. Rev. D 96, 115012 (2017).

[30] R. Barbieri, L. J. Hall, and V. S. Rychkov, Phys. Rev. D 74, 015007 (2006).

[31] D. S. Akerib et al. (LUX Collaboration), Phys. Rev. Lett. 118, 021303 (2017).

[32] E. Aprile et al. (XENON Collaboration), Phys. Rev. Lett. 119, 181301 (2017).

[33] V. Khachatryan et al. (CMS Collaboration), J. High Energy Phys. 12 (2016) 013.

[34] M. Aaboud et al. (ATLAS Collaboration), J. High Energy Phys. 06 (2018) 107.

[35] M. Gustafsson, S. Rydbeck, L. Lopez-Honorez, and E. Lundstrom, Phys. Rev. D 86, 075019 (2012).

[36] P. Poulose, S. Sahoo, and K. Sridhar, Phys. Lett. B 765, 300 (2017).

[37] A. Datta, N. Ganguly, N. Khan, and S. Rakshit, Phys. Rev. D 95, 015017 (2017).

[38] E. W. Kolb and M. S. Turner, Frontiers in Physics (Westview Press, Colorado, 1990).

[39] R. J. Scherrer and M. S. Turner, Phys. Rev. D 33, 1585 (1986); 34, 3263(E) (1986).

[40] G. Jungman, M. Kamionkowski, and K. Griest, Phys. Rep. 267, 195 (1996).

[41] P. Gondolo and G. Gelmini, Nucl. Phys. B360, 145 (1991).

[42] K. Griest and D. Seckel, Phys. Rev. D 43, 3191 (1991).
[43] E. Lundstrom, M. Gustafsson, and J. Edsjo, Phys. Rev. D 79, 035013 (2009).

[44] J. P. Leveille, Nucl. Phys. B137, 63 (1978).

[45] G. Bhattacharyya, K. B. Chatterjee, and S. Nandi, Nucl. Phys. B831, 344 (2010).

[46] M. Lindner, M. Platscher, and F. S. Queiroz, Phys. Rep. 731, 1 (2018).

[47] W. Altmannshofer, P. Ball, A. Bharucha, A. J. Buras, D. M. Straub, and M. Wick, J. High Energy Phys. 01 (2009) 019.

[48] D. Becirevic, N. Kosnik, F. Mescia, and E. Schneider, Phys. Rev. D 86, 034034 (2012).

[49] A. J. Buras and M. Munz, Phys. Rev. D 52, 186 (1995).

[50] C. Bobeth, M. Gorbahn, T. Hermann, M. Misiak, E. Stamou, and M. Steinhauser, Phys. Rev. Lett. 112, 101801 (2014).

[51] R. Aaij et al. (LHCb Collaboration), Phys. Rev. Lett. 118, 191801 (2017).

[52] G. Hiller and M. Schmaltz, Phys. Rev. D 90, 054014 (2014).

[53] R. Alonso, B. Grinstein, and J. Martin Camalich, Phys. Rev. Lett. 113, 241802 (2014).

[54] A. Lenz and U. Nierste, J. High Energy Phys. 06 (2007) 072.

[55] L. F. Abbott, P. Sikivie, and M. B. Wise, Phys. Rev. D 21, 1393 (1980).

[56] J. Charles, S. Descotes-Genon, Z. Ligeti, S. Monteil, M. Papucci, and K. Trabelsi, Phys. Rev. D 89, 033016 (2014).

[57] S. Aoki et al. (Flavour Lattice Averaging Group), arXiv: 1902.08191.

[58] J. Charles et al., Phys. Rev. D 91, 073007 (2015).

[59] J. Aebischer, W. Altmannshofer, D. Guadagnoli, M. Reboud, P. Stangl, and D. M. Straub, arXiv:1903.10434.

[60] P. Achard et al. (L3 Collaboration), Phys. Lett. B 517, 75 (2001).

[61] A. Alloul, N. D. Christensen, C. Degrande, C. Duhr, and B. Fuks, Comput. Phys. Commun. 185, 2250 (2014).

[62] J. Alwall, R. Frederix, S. Frixione, V. Hirschi, F. Maltoni, O. Mattelaer, H.-S. Shao, T. Stelzer, P. Torrielli, and M. Zaro, J. High Energy Phys. 07 (2014) 079.

[63] T. Sjostrand, S. Mrenna, and P. Z. Skands, J. High Energy Phys. 05 (2006) 026.

[64] R. Placakyte, arXiv:1111.5452.

[65] A. M. Sirunyan et al. (CMS Collaboration), J. High Energy Phys. 05 (2018) 025.

[66] F. Lyonnet and I. Schienbein, Comput. Phys. Commun. 213, 181 (2017).

[67] N. Chakrabarty, D. K. Ghosh, B. Mukhopadhyaya, and I. Saha, Phys. Rev. D 92, 015002 (2015). 